\title{
Richtungsfelder und
}

Fernparallelismus in $\mathrm{n}$ -

\section{dimensionalen Mannigfaltigkeiten}

\section{Doctoral Thesis}

Author(s):

Stiefel, Eduard

Publication date:

1935

Permanent link:

https://doi.org/10.3929/ethz-a-000092403

Rights / license:

In Copyright - Non-Commercial Use Permitted 


\title{
Richtungsfelder und \\ Fernparallelismus in $\mathbf{n}$-dimensionalen Mannigfaltigkeiten
}

\author{
Von der \\ Eidgenössischen Technischen Hochschule in Zürich \\ zur Erlangung der Würde eines Doktors der Mathematik \\ genehmigte \\ Promotionsarbeit \\ vorgelegt von \\ EDUARD STIEFEL \\ aus Zürich
}

Referent: Herr Prof. Dr. H. Hopf

Korreferent: Herr Prof. Dr. F. Gonseth

$\begin{array}{llll}1 & 9 & 3 & 5\end{array}$

ART. INSTITUT ORELLFÖSSLI, ZÜRICH 
Sonderabdruck aus den

Commentarii Mathematici Helvetici, vol. 8, fasc. 4, 1935/36 


\section{Einleitung}

1. Die $n$-dimensionalen Mannigfaltigkeiten, welche in dieser Arbeit betrachtet werden, sollen geschlossen und stetig differenzierbar $\operatorname{sein}^{1}$ ). Die Frage, ob in einer solchen Mannigfaltigkeit ein ausnahmslos stetiges Richtungsfeld existiert, wird durch den folgenden bekannten Satz beantwortet $\left.{ }^{2}\right)$ :

Satz $A_{1}$. Dann und nur dann existiert in der Mannigfaltigkeit $M^{n}$ ein singularitätenfreies stetiges Richtungsfeld, wenn die Euler'sche Charakteristik von $M^{n}$ den Wert 0 hat $(\xi 5, N r .2)$.

Daher sind einerseits unter allen geschlossenen und orientierbaren Flächen diejenigen vom topologischen Typus des Torus die einzigen, welche die Anbringung eines stetigen Richtungsfeldes erlauben ${ }^{3}$ ); andererseits kann man jede Mannigfaltigkeit ungerader Dimensionszahl, insbesondere also jede dreidimensionale Mannigfaltigkeit, mit einem stetigen Richtungsfeld ausstatten ( $\$ 6, \mathrm{Nr} .1$ ).

Da aber nicht zu erwarten ist, daß sich alle Mannigfaltigkeiten ungerader Dimensionszahl bezüglich der in ihnen existierenden stetigen Richtungsfelder ganz gleichartig verhalten, wird man durch den soeben formulierten Gegensatz (z. B. zwischen $n=2$ und $n=3$ ) veranlaßt, eine Verfeinerung der ursprünglichen Frage zu suchen. Es liegt nahe, folgendermaßen zu fragen: Eine $n$-dimensionale Mannigfaltigkeit $M^{n}$ und eine Zahl $m$ aus der Reihe $1,2, \ldots, n$ seien vorgelegt; gibt es in $M^{n}$ ein System von $m$ stetigen Richtungsfeldern, welche in jedem Punkt der $M^{n}$ linear unabhängig sind?

Diese Frage, die für $m=1$ durch den Satz $A_{1}$ beantwortet wird, und die besonderes und selbständiges Interesse für $m=n-1$ und $m=n$ beanspruchen darf (vgl. Nr. 5 dieser Einleitung), bildet den Gegenstand

1) Man vgl. Kap. XIV, § 4, der „Topologie“ (1. Band) von Alexandroff und Hopf. (J. Springer, Berlin, 1935.) Dieses Buch, dessen Terminologie wir in dieser Arbeit folgen, wird im folgenden kurz als „AH“" zitiert.

2) AH: Kap. XIV, § 4, Satz III.

3) Poincaré: Journal de Liouville, 4e série, t. I., p. 203-208. 
der vorliegenden Arbeit. Die Frage wird zwar nicht in dem Sinne vollständig beantwortet, daß, in Verallgemeinerung des Satzes $A_{1}$, eine notwendige und hinreichende Bedingung für die Existenz eines Systemes von $m$ unabhängigen Richtungsfeldern - im folgenden kurz , $m$-Feld“ genannt - aufgestellt wird; jedoch werden einige Sätze bewiesen, welche einerseits das Problem in manchen Spezialfällen zu erledigen gestatten, und welche andererseits neue Beiträge zur allgemeinen Topologie der geschlossenen Mannigfaltigkeiten darstellen.

2. Bevor wir die wichtigsten dieser Sätze formulieren, erinnern wir an den mit dem Satz $A_{1}$ verwandten und ebenfalls bekannten ${ }^{4}$ )

Satz $B_{1}$. In jeder Mannigfaltigkeit $M^{n}$ gibt es Richtungsfelder, welche in höchstens endlich vielen Punkten singulär (d.h. unstetig) werden; die Anzahl dieser Singularitäten, mit richtigen Vielfachheiten (,Indexen") gezählt, ist unabhängig von dem speziellen Felde: sie ist stets gleich der Charakteristik von $M^{n}(\S 5, N r .2)$.

Von diesem Satz werden wir die folgende Verallgemeinerung beweisen:

Satz $B_{m}$. In jeder $M^{n}$ gibt es für jedes $m(1 \leqq m \leqq n) m$-Felder, deren Singularitäten (d.h. Unstetigkeitspunkte der einzelnen Richtungsfelder oder Punkte linearer Abhängigkeit der verschiedenen Felder) einen höchstens ( $m-1)$-dimensionalen Komplex bilden; bei richtiger Zählung der Vielfachheiten der Singularitäten ist er ein Zyklus, und die Homologielklasse dieses Zyklus ist unabhängig von dem speziellen $m$-Feld: sie ist ein ausgezeichnetes Element der (m-1)-ten Betti'schen Gruppe $\left.e^{4 a}\right)$ von $M^{n}(\$ 4, N r .4,5)$.

Diese Homologieklasse $F^{m-1}$ nennen wir die ,m-te charalteristische Klasse" von $M^{n}$; im Fall $m=1$ ist sie diejenige nulldimensionale Homologieklasse, in welcher sich ein mit der Euler'schen Charakteristik multiplizierter Punkt von $M^{n}$ befindet.

Der Satz $A_{1}$ wird nun in gewissem Sinne durch den folgenden Satz verallgemeinert:

Satz $A_{m}$. Dann und nur dann gibt es in $M^{n}$ ein $m$-Feld, dessen Singularitäten einen höchstens $(m-2)$-dimensionalen Komplex bilden, wenn $F^{m-1}=0$ (d.h. das Nullelement der $(m-1)$-ten Betti'schen Gruppe von $\left.M^{n}\right)$ ist $(\S 4, N r .5)$.

\footnotetext{
4) AH: Kap. XIV, § 4, Satz I.

4a) Der Koeffizientenbereich, auf den sich diese Betti'sche Gruppe bezieht, ist in § 4, Nr. 3 definiert (Vgl. auch AB : Kap. V).
} 
Hieraus folgt sofort:

Satz $A_{m}^{\prime}$. Für die Existenz eines singularitätenfreien $m$-Feldes in $M^{n}$ ist notwendig, daß

ist.

$$
F^{0}=F^{1}=\cdots=F^{m-1}=0
$$

Hinreichend dürfte diese Bedingung jedoch nicht sein.

3. Nun liegt die Aufgabe nahe, für eine vorgelegte $M^{n}$ die charakteristischen Klassen $F^{m-1}(m=1,2 \ldots)$ zu bestimmen. Im Fall $m=1$ ist die Bestimmung von $F^{m-1}$ gleichbedeutend mit der Bestimmung der Euler'schen Charakteristik von $M^{n}$, und diese kann man auf Grund der Euler-Poincaré'schen Formel

$$
\Sigma(-1)^{r} \alpha^{r}=\Sigma(-1)^{r} p^{r},
$$

wobei $a^{r}$ die Anzahl der $r$-dimensionalen Zellen einer Zerlegung von $M^{n}$, $p^{r}$ die $r$-te Betti'sche Zahl von $M^{n}$ bezeichnet, auf zweierlei Weisen ausdrücken: erstens durch die $a^{r}$, zweitens durch die $p^{r}$.

Die erste dieser beiden Möglichleiten scheint sich auf beliebiges $m$ übertragen zu lassen ( $\$ 5, \mathrm{Nr} .3$, Fußnote 22); prinzipiell wichtiger ist aber die Frage, ob man die Klasse $F^{m-1}$ auch auf eine Weise darstellen kann, welche der Darstellung der Charakteristik durch die rechte Seite der Euler-Poincaré'schen Formel entspricht, also durch bekannte topologische Invarianten von $M^{n}$. Auch wenn die, uns bisher unbekannte Antwort auf diese Frage verneinend wäre, würde sie doch etwas Neues lehren: $F^{m-1}$ wäre eine neue topologische Invariante von Mannigfaltigkeiten.

Noch in anderer Hinsicht besteht eine Beziehung zwischen der Klasse $F^{m-1}$ und der Euler'schen Charakteristik: die Schnittzahl von $F^{m-1}$ mit einer in die $M^{n}$ eingebetteten $(n-m+1)$-dimensionalen Mannigfaltigkeit ist $(\bmod 2)$ der Charakteristik dieser Mannigfaltigkeit kongruent, falls die Einbettung gewisse, in $\S 6, \mathrm{Nr} .2$ formulierte Forderungen erfüllt.

4. Die Bestimmung von $F^{m-1}$ für eine vorgelegte Mannigfaltigkeit gelingt unter Umständen allein mit Hilfe des Satzes $B_{m}$; denn auf Grund dieses Satzes braucht man ja nur ein spezielles $m$-Feld zu konstruieren, welches so übersichtlich gebaut ist, daß man den Komplex seiner Singularitäten beherrscht. Auf diese Weise werden wir als Beispiel die $(4 k+1)$ dimensionalen projektiven Räume behandeln; es wird sich zeigen: 
Satz $C$. Für den $(4 k+1)$-dimensionalen reellen projektiven Raum $P^{4 k+1}$ ist $F^{1}$ diejenige Klasse, welche die projektive Gerade enthält, sie ist also nicht Null (\$6, Nr.3).

Hierin und in der Tatsache, daß es in jeder Mannigfaltigkeit ungerader Dimensionszahl ein stetiges Richtungsfeld gibt, ist enthalten:

Satz $C^{\prime}$. Im $P^{4 k+1}$ gibt es stetige Richtungsfelder, jedoch existieren für jedes Paar von Feldern Punkte, in welchen die Richtungen der beiden Felder entweder gleich- oder entgegengesetzt gerichtet sind.

Diese Eigenschaft projektiver Räume gestattet den Nachweis gewisser algebraischer Sätze, für welche bisher keine Beweise bekannt zu sein scheinen, die mit den üblichen algebraischen Hilfsmitteln arbeiten $(\S 6, \mathrm{Nr} .3)$.

5. Besonderes und selbständiges Interesse verdient die Frage, ob es in einer $M^{n}$ ein $n$-Feld gibt; die Existenz eines solches Feldes ist nämlich gleichbedeutend damit, daß man in $M^{n}$ einen Fernparallelismus einführen kann oder, wie wir auch sagen, daß $M^{n}$,parallelisierbar" ist. Dabei nennen wir $M^{n}$ parallelisierbar, wenn man die Gesamtheit aller Richtungen in $M^{n}$ derart in zueinander fremde, eindeutige und stetige Richtungsfelder, die wir ,Parallelenfelder" nennen, zerlegen kann, daß die folgende Bedingung erfüllt ist: Sind $\mathfrak{v}_{1}, \mathfrak{v}_{2}, \ldots, \mathfrak{v}_{k}$ Richtungen in einem Punkt $p$ von $M^{n}$ und $\mathfrak{v}_{1}^{\prime}, \mathfrak{v}_{2}^{\prime}, \ldots, \mathfrak{v}_{k}^{\prime}$ die denselben Parallelenfeldern entnommenen Richtungen in einem beliebigen anderen Punkt $p^{\prime}$, so folgt aus der linearen Unabhängigkeit der $\mathfrak{v}_{i}$ die lineare Unabhängigkeit der $\mathfrak{\mathfrak { v }}_{i}^{\prime}$. Wir werden Richtungen, die demselben Parallelenfeld entnommen sind, kurz ,parallel" nennen.

Man sieht in der Tat leicht, daß die Parallelisierbarkeit mit der Existenz eines $n$-Feldes identisch ist: Wenn ein $n$-Feld existiert, so nenne man zwei Richtungen $\mathfrak{v}, \mathfrak{v}^{\prime}$ in den Punkten $p$ bezw. $p^{\prime}$ einander ,parallel“", falls ihre Komponenten in bezug auf die in $p$ bezw. $p^{\prime}$ angebrachten Richtungen des $n$-Feldes bis auf einen positiven Faktor miteinander übereinstimmen; dann hat man einen Fernparallelismus eingeführt. Ist andererseits ein Fernparallelismus definiert, so zeichne man in einem festen Punkt $n$ linear unabhängige Richtungen aus; die zu diesen Richtungen parallelen Richtungen in den übrigen Punkten von $M^{n}$ bilden dann ein $n$-Feld.

Nichtorientierbare Mannigfaltigkeiten sind nicht parallelisierbar; andererseits zeigt man leicht, da $\beta$ in einer orientierbaren Mannigfaltigkeit die Existenz eines $n$-Feldes bereits aus der Existenz eines $(n-1)$ Feldes folgt. Damit ist die Untersuchung der Parallelisierbarkeit voll- 
ständig auf die Untersuchung der $(n-1)$-Felder zurückgeführt; es bedeutet also keine Einschränkung, wenn wir im folgenden $m<n$ voraussetzen. Aus Satz $A_{m}^{\prime}$ ergibt sich:

Satz D. Notwendig für die Parallelisierbarkeit von $M^{n}$ ist das Verschwinden aller charalteristischen Klassen $F^{0}, F^{1}, \ldots, F^{n-2}$.

Auch hier - vergleiche Satz $A^{\prime}{ }_{m}$ - ist nicht anzunehmen, daß die Bedingung hinreicht.

Da eine Gruppenmannigfaltigkeit ${ }^{5}$ ) sicher parallelisierbar ist, ergibt Satz $D$ eine notwendige Bedingung dafür, daß die vorgelegte Mannigfaltigkeit $M^{n} \mathrm{zu}$ einem Aruppenraum gemacht werden kann.

6. Nichtparallelisierbar sind gewiß alle Mannigfaltigkeiten, für welche die Euler'sche Charakteristik $\neq 0$ ist, also z. B. die Sphären gerader Dimension, denn dann ist ja auch $F^{0} \neq 0$; ferner die in Satz $C$ genannten projektiven Räume der Dimension $4 k+1$. Durch Produktbildung kann man ferner beweisen:

Satz E. Für jede von 1 und 3 verschiedene Dimensionszahl n gibt es n-dimensionale (geschlossene und orientierbare) Mannigfaltigkeiten, die nicht parallelisierbar sind $(\S 6, N r, 2)$.

Für $n=1$ gibt es eine einzige geschlossene Mannigfaltigkeit: den Kreis; er ist trivialerweise parallelisierbar. Offen ist die Frage der Parallelisierbarkeit daher zunächst nur noch für $n=3$; und hier gilt:

Satz F. Jede dreidimensionale geschlossene und orientierbare Mannigfaltigkeit $^{5 \text { a }}$ ) ist parallelisierbar ( $\left.\$ 5, N r .3\right)$.

Diese merkwürdige Ausnahmestellung der Dimensionszahl 3 zeigt von neuem die Schwierigkeit bei den Klassifikationsversuchen für die dreidimensionalen Mannigfaltigkeiten: der Versuch, die orientierbaren dreidimensionalen Mannigfaltigkeiten in parallelisierbare und nicht-parallelisierbare einzuteilen, mißlingt.

7. Die in dieser Einleitung aufgestellten Sätze werden in den $\S \S 4-6$ formuliert und bewiesen; die $\S \S 1$ und 2 haben vorbereitenden Charakter. Von $\S 1$ sind nur die Definitionen in Nr. 1 und die Resultate in Nr. 4 für den übrigen Teil der Arbeit wichtig. Im Anhang I wird die Bestimmung der Klasse $F^{1}$ in dreidimensionalen orientierbaren Mannigfaltigkeiten nachgeholt, die in $\S 5, \mathrm{Nr} .3$ nur angedeutet wurde. Der Anhang II ist

5) AH: Einleitung $\S 3$, Nr. 17; dort finden sich auch Literaturangaben.

5 a) Die Mannigfaltigkeit muß überdies gewisse Differenzierbarkeits-Voraussetzungen erfüllen (vgl. $\$ 5$, Nr. 3, und Anhang I). 
nachträglich entstanden; in ihm wird bewiesen, daß eine im euklidischen Raum liegende Mannigfaltigkeit mit ungerader Charakteristik nicht durch Gleichungen regulär dargestellt werden kann ${ }^{6}$ ).

Über einzelne Teilresultate aus dieses Arbeit habe ich bereits an anderer Stelle (Verh. der schw. naturf. Gesellschaft 1934, S. 270; ferner: Enseignement mathématique, 1934, 1, p. 6) berichtet.

lch möchte an dieser Stelle Herrn Prof. H. Hopf für die Anregung zu dieser Arbeit, für sein dauerndes Interesse an ihren Fortschritten, sowie für seinen wertvollen Rat in entscheidenden Momenten danken.

\section{§ 1. Die Mannigfaltigkeiten $V_{n, m}$.}

\section{Definitionen}

Ein geordnetes und normiertes Orthogonalsystem $\sigma_{n, m}$ von $m$ Vektoren $\mathfrak{v}_{1}, \mathfrak{v}_{2} \ldots \mathfrak{v}_{m}$, die in einem Punkt des euklidischen $n$-dimensionalen Raumes $R^{n}$ angreifen, nennen wir im folgenden ein $m$-System des $R^{n}$. Dabei sei $m$ an die Ungleichungen

$$
0<m<n
$$

gebunden. $V_{n, m}$ ist definiert als die Menge aller in einem festen Punkt des $R^{n}$ angreifenden $m$-Systeme $\sigma_{n, m}$; führt man auf naheliegende Weise in dieser Menge einen Umgebungsbegriff ein, so wird $V_{n, m}$ zu einem topologischen Raum, dessen Punkte $v$ die $m$-Systeme $\sigma_{n, m}$ sind.

$V_{n, 1}$ ist zu der $(n-1)$-dimensionalen Sphäre $S^{n-1}$, die vom Endpunkt des Vektors $\mathfrak{y}_{1}$ durchlaufen wird, homöomorph. Ist aber $m>1$, so verschieben wir die Vektoren $\mathfrak{v}_{2} \ldots \mathfrak{b}_{m}$ von $\sigma_{n, m}$ parallel an den Endpunkt des Vektors $\mathfrak{v}_{1} . \quad V_{n, m}$ kann also auch als die Menge aller an die $S^{n-1}$ tangentialen $(m-1)$-Systeme $\sigma_{n, m-1}$ des $R^{n}$ erklärt werden. Speziell ist $V_{n, 2}$ die Menge der gerichteten Linienelemente der $S^{n-1}$.

Durch stereographische Projektion kann man eine weitere Darstellung des Raumes $V_{n, m}$, den wir im folgenden kurz mit $V$ bezeichnen, gewinnen: Projiziert man die $S^{n-1}$ von ihrem Nordpol aus auf ihren Äquatorialraum $R^{n-1}$, so geht ein im Sphärenpunkt $p$ angreifendes System $\sigma_{n, m-1}$ in ein im Bildpunkt $p_{1}$ von $p$ angreifendes $(m-1)$ System $\sigma_{n-1, m-1}$ des $R^{n-1}$ über. $\sigma_{n-1, m-1}$ ist eindeutig festgelegt durch seinen Angriffspunkt $p_{1}$ und das zu $\sigma_{n-1, m-1}$ parallele $(m-1)$-System einer in den $R^{n-1}$ eingebetteten $V_{n-1, m-1}=V_{1}^{\prime}$. Ein Punkt $v$ von $V$

6) Man vgl. auch AH: Einleitung, § 1, Nr. 7. 
ist somit gegeben durch einen Punkt $p_{1}$ des $R^{n-1}$ und einen Punkt $v_{1}$ von $V_{1}^{\prime}$. Wir schreiben kurz:

$$
v=p_{1} \times v_{1}
$$

Diese Darstellung versagt nur für diejenigen Systeme $\sigma_{n, m-1}$, die im Nordpol angreifen. Um auch diese Systeme zu erfassen, projizieren wir die $S^{n-1}$ noch vom Südpol aus auf den $R^{n-1}$. Analog zu (2) erhält man:

$$
v=p_{2} \times v_{2} .
$$

$v_{2}$ ist ein Punkt der Menge $V_{2}^{\prime}$, die bei der zweiten Projektion an Stelle von $V_{1}^{\prime}$ tritt. Bezeichnen wir die Äquatorialsphäre der $S^{n-1}$ mit $S^{n-2}$, so gehen die beiden Punkte $p_{1}$ und $p_{2}$ durch die Transformation vermittelst reziproker Radien an der $S^{n-2}$ auseinander hervor.

Die Formel (2) stellt eine Beziehung zwischen $V$ und $V_{1}^{\prime}$, d. h. zwischen $V_{n, m}$ und $V_{n-1, m-1}$ her. Durch Iteration erhalten wir eine Beziehung zwischen den Räumen der Reihe:

$$
V_{n, m}, V_{n-1, m-1}, \ldots V_{n-k, m-k}, \ldots V_{n-m+1,1}=S^{n-m} .
$$

Daraus lassen sich folgende Schlüsse ziehen:

I. Jeder Punkt von $V_{n, m}$ besitzt eine zum Innern einer euklidischen Vollkugel homöomorphe Umgebung.

II. $V_{n, m}$ ist zusammenhängend. (Wegen (1) ist $S^{n-m}$ zusammenhängend.)

III. Für die Dimension $\mu_{n, m}$ von $V_{n, m}$ gilt die Rekursionsformel

$$
\mu_{n, m}=\mu_{n-1, m-1}+(n-1) \text {, }
$$

also

$$
\mu_{n, m}=m \cdot\left(n-\frac{m+1}{2}\right)
$$

2. Zerlegung der $V_{n, m}$

In unserer ersten Projektion begrenze $S^{n-2}$ die im $R^{n-1}$ liegende abgeschlossene Vollkugel $E_{1}$. Wir definieren:

$$
K_{1}=E_{1} \times V_{1}^{\prime}
$$


Analog in der zweiten Projektion:

$$
K_{2}=E_{2} \times V_{2}^{\prime} \text {. }
$$

Dann ist $V$ die Vereinigungsmenge von $K_{1}$ und $K_{2}$ :

$$
V=K_{1}+K_{2} \text {. }
$$

Iteriert man diese Zerlegung der $V_{n, m}$ in der Reihe (4), so folgt auf induktivem Wege das Resultat

IV. $V_{n, m}$ ist ein Polyeder.

Aus I-IV folgt nun:

Satz 1. $V_{n, m}$ ist eine geschlossene Mannigfaltigkeit.

Die Mannigfaltigkeiten der Reihe (4) nennen wir die der $V_{n, m}$ zugeordneten Mannigfaltigkeiten.

Für den Durchschnitt von $K_{1}$ und $K_{2}$ ergibt sich:

In der ersten Projektion: $K_{1} \cdot K_{2}=S^{n-2} \times V_{1}^{\prime}$,

In der zweiten Projektion: $K_{1} \cdot K_{2}=S^{n-2} \times V_{2}^{\prime}$.

Aus unserer Zerlegung (9) der Mannigfaltigkeit $V$ wollen wir durch Induktion in der Reihe der zugeordneten Mannigfaltigkeiten Eigenschaften der Betti'schen Gruppen von $V$ herleiten. Wir verstehen unter $B^{r}(K)$ für $r>0$ die $r$-dimensionale ganzzahlige Betti'sche Gruppe des Komplexes $K$, für $r=0$ die Gruppe der 0 -dimensionalen ganzzahligen Homologieklassen, die nur berandungsfähige Zyklen enthalten. (Ein 0-dimensionaler Zyklus ist berandungsfähig, wenn die Summe seiner Koeffizienten verschwindet ${ }^{7}$ ). Algebraische Teilkomplexe von

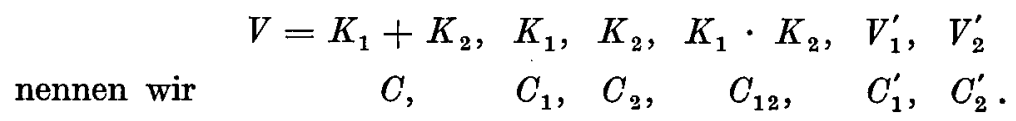

Zyklen werden immer mit $z$ oder $Z$ bezeichnet.

Nun legen wir die folgende Voraussetzung zugrunde:

$$
\begin{gathered}
\text { für ein festes } r \text { mit } 0 \leqq r<n-2 \\
\text { sei } B^{r}\left(V_{n-1, m-1}\right)=0 .
\end{gathered}
$$

7) AH: Kap. IV, § 4, Nr. 7 ferner Kap. V, § 1, Nr. 5. 
Dann gilt $\left.{ }^{8}\right)$ für einen beliebigen $(r+1)$-dimensionalen Teilzyklus $z^{r+1}$ von $V_{n, m}$ :

$$
z^{r+1}=z_{1}^{r+1}+z_{2}^{r+1}
$$

$\left(z_{1}^{r+1}\right.$ ist Teilzyklus von $K_{1}$ und $z_{2}^{r+1}$ Teilzyklus von $\left.K_{2}\right)$.

Beweis : Aus $\left(J_{1}\right)$ folgt zunächst $B^{r}\left(V_{1}^{\prime}\right)=0$, also ist $\left.\operatorname{auch}^{9}\right)$ $B^{r}\left(S^{n-2} \times V_{1}^{\prime}\right)=0$, und somit nach (10):

$$
B^{r}\left(K_{1} \cdot K_{2}\right)=0
$$

Sei nun $z^{r+1}=C_{1}-C_{2}$ irgend eine Zerlegung von $z^{r+1}$ in zwei algebraische $(r+1)$-dimensionale Teilkomplexe von $K_{1}$ und $K_{2}$. Durch Randbildung ergibt sich $\dot{C}_{1}=\dot{C}_{2}$; dieser gemeinsame Rand liegt sowohl in $K_{1}$ als auch in $K_{2}$, ist also ein $z_{12}^{r}$. Aus (13) folgt $z_{12}^{r}=\dot{C}_{12} . C_{1}-C_{12}, C_{2}-C_{12}$ sind Zyklen $z_{1}, z_{2}$, und es ist $z^{r+1}=z_{1}-z_{2}$, womit (12) bewiesen ist.

Unter der schärferen Voraussetzung:

für ein festes $r$ mit $0 \leqq r<n-3$

$$
\text { sei } B^{r}\left(V_{n-1, m-1}\right)=0
$$

erhält man noch den Isomorphismus:

$$
B^{r+1}\left(V_{n, m}\right) \approx B^{r+1}\left(V_{n-1, m-1}\right)
$$

Beweis: Aus den Sätzen über die Betti'schen Gruppen von Produktkomplexen folgt:

$$
B^{r+1}\left(K_{1}\right)=B^{r+1}\left(E_{1} \times V_{1}^{\prime}\right)=B^{r+1}\left(E_{1} \times V_{n-1, m-1}\right) \approx B^{r+1}\left(V_{n-1, m-1}\right) .
$$

Analog erhält man unter Berücksichtigung von $r+1<n-2$ :

$$
B^{r+1}\left(K_{1} \cdot K_{\dot{2}}\right)=B^{r+1}\left(S^{n-2} \times V_{1}^{\prime}\right)=B^{r+1}\left(S^{n-2} \times V_{n-1, m-1}\right) \approx B^{r+1}\left(V_{n-1, m-1}\right),
$$

und daher

$$
B^{r+1}\left(K_{1} \cdot K_{2}\right) \approx B^{r+1}\left(K_{1}\right)
$$

Dieser Isomorphismus kann realisiert werden, indem man einer Homologieklasse von $K_{1} \cdot K_{2}$ mit dem repräsentierenden Zyklus $z_{12}^{r+1}$ die

8) Dieser Satz ist Spezialfall eines Additionssatzes der kombinatorischen Topologie; vgl. AH: Kap. VII, § 2, speziell Nr. 5.

9) Utber Betti'sche Gruppen von Produktkomplexen vgl. AH: Kap. VII, § 3. 
Homologieklasse von $z_{12}^{r+1}$ in $K_{1}$ zuordnet. Daraus ziehen wir die folgenden Schlüsse:

a) In jeder $(r+1)$-dimensionalen Homologieklasse von $K_{1}$ (oder $K_{2}$ ) ist ein Zyklus von $K_{1} \cdot K_{2}$ enthalten.

b) Aus der Homologie $z_{12}^{r+1} \sim 0$ in $K_{1}$ (oder $K_{2}$ ) folgt

$$
z_{12}^{r+1} \sim 0 \text { in } K_{1} \cdot K_{2} \text {. }
$$

Ordnet man nun einer Homologieklasse von $K_{1} \cdot K_{2}$ mit dem repräsentierenden Zyklus $Z_{12}^{r+1}$ die Homologieklasse von $Z_{12}^{r+1}$ in $K_{1}+K_{2} z u$, so entsteht eine homomorphe Abbildung von $B^{r+1}\left(K_{1} \cdot K_{2}\right)$ in $B^{r+1}\left(K_{1}+K_{2}\right)$. Diese Abbildung ist ein Isomorphismus, falls

1. in jeder $(r+1)$-dimensionalen Homologieklasse von $K_{1}+K_{2}$ ein Zyklus von $K_{1} \cdot K_{2}$ enthalten ist,

2. aus $Z_{12}^{r+1} \sim 0$ in $K_{1}+K_{2}$ die Homologie $Z_{12}^{r+1} \sim 0$ in $K_{1} \cdot K_{2}$ folgt.

1. folgt aus (12) und a); 2. wird auf die folgende Weise verifiziert: $Z_{12}^{r+1} \sim 0$ in $K_{1}+K_{2}$ heißt $Z_{12}^{r+1}=\dot{C}$. Eine Zerlegung $C=C_{1}-C_{2}$ von $C$ liefert $Z_{12}^{r+1}=\dot{C}_{1}-\dot{C}_{2}$. Dies ist nur möglich, wenn $\dot{C}_{1}=z_{12}^{r+1}$ und $\dot{C}_{2}=\bar{z}_{12}^{r+1}$. Da $z_{12}^{r+1} \sim 0$ in $K_{1}$, ergibt sich aus b) $z_{12}^{r+1} \sim 0$ in $K_{1} \cdot K_{2}$, und ebenso $\bar{z}_{12}^{r+1} \sim 0$ in $K_{1} \cdot K_{2}$, also auch $Z_{12}^{r+1} \sim 0$ in $K_{1} \cdot K_{2}$. Somit haben wir $B^{r+1}\left(K_{1}+K_{2}\right) \approx B^{r+1}\left(K_{1} \cdot K_{2}\right)$. Die Formel (15) beweist dann die Behauptung (14). Aus unserem Beweis ergibt sich noch der

Hilfssatz: Unter der Voraussetzung $\left(J_{2}\right)$ ist eine $(r+1)$-dimensionale Homologiebasis in $K_{1} \cdot K_{2}$ auch eine Homologiebasis für $V=K_{1}+K_{2}$.

Jetzt läßt sich leicht der folgende Satz beweisen:

Satz 2. Für $0 \leqq r<n-m-1$ ist $B^{r+1}\left(V_{n, m}{ }^{\prime}\right)=0$.

Der Beweis erfolgt durch vollständige Induktion in der Reihe der zugeordneten Mannigfaltigkeiten; es sei also bereits bewiesen:

$$
B^{r+1}\left(V_{n-1, m-1}\right)=0 \text { für } 0 \leqq r<n-m-1 .
$$

Aus Satz 1 folgt ferner $B^{0}\left(V_{n-1, m-1}\right)=0$, also ist auch $B^{r}\left(V_{n-1, m-1}\right)=0$. Da $m>1$ vorausgesetzt war, gilt $\left(J_{2}\right)$ und somit (14) und daher Satz 2. Die Induktion wird bei der Mannigfaltigkeit $V_{n-m+1,1}=S^{n-m}$ verankert, für die Satz 2 trivial ist. 
Satz 3. Für $m>2$ ist $B^{n-m}\left(V_{n, m}\right) \approx B^{n-m}\left(V_{n-1, m-1}\right)$.

Beweis: Nach Satz 2 gilt $\left(J_{2}\right)$ für $r=n-m-1$. (14) liefert die Behauptung.

\section{Topologie der $V_{n, 2}$.}

Aus Satz $3 \mathrm{kann} B^{n-m}\left(V_{n, m}\right)$ bestimmt werden, wenn $B^{n-m}\left(V_{n-m+2,2}\right)$ bekannt ist; es soll daher in diesem Abschnitt zunächst die $(n-2)$ dimensionale Betti'sche Gruppe einer Mannigfaltigkeit $V_{n, 2}$ berechnet werden. Die Reihe der zugeordneten Mannigfaltigkeiten besteht in diesem Fall einzig aus einer $(n-2)$-dimensionalen Sphäre. Zur Darstellung der $V_{n, 2}$ benützen wir unsere erste Projektion; dann ist also $V_{1}^{\prime}$ eine Sphäre $S_{1}^{\prime n-2}$. Die beiden Sphären $S^{n-2}$ und $S_{1}^{\prime n-2}$ seien gleich orientiert, die durch diese Orientierungen gelieferten Zyklen bezeichnen wir auch mit $S^{n-2}$ und $S_{1}^{\prime n-2}$. Ist $s$ ein beliebiger aber fest gewählter Punkt der $S^{n-2}$ und $s_{1}^{\prime}$ ein Punkt der $S_{1}^{\prime n-2}$, so bilden wegen (10) die beiden Zyklen $z_{12}=s \times S_{1}^{\prime n-2}$ und $\bar{z}_{12}=S^{n-2} \times s_{1}^{\prime}$ eine $(n-2)$-dimensionale Homologie-

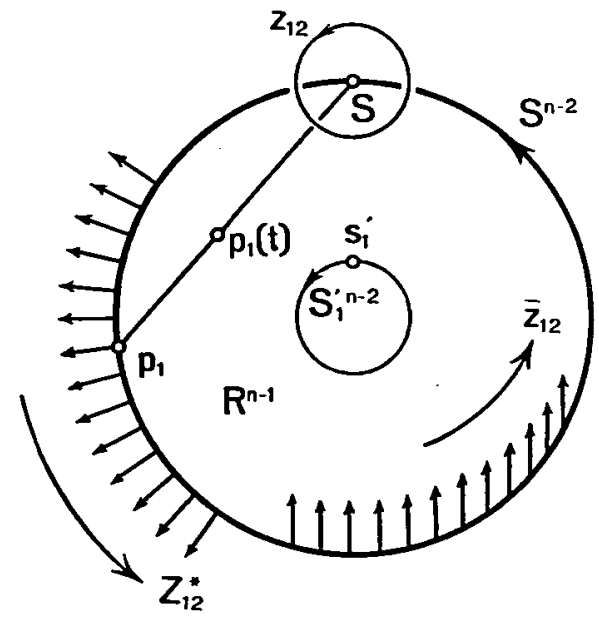

Fig 1.

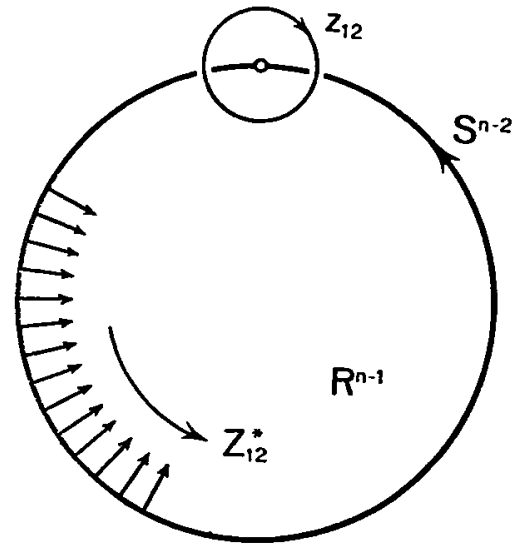

Fig 2.

basis in $K_{1} \cdot K_{2}$. (In Fig. 1 ist der Fall $n=3$ dargestellt.) Irgend ein $(n-2)$-dimensionaler Zyklus $Z_{12}$ von $K_{1} \cdot K_{2}$ erfüllt also eine Homologie

$$
Z_{12} \sim \alpha z_{12}+\beta \bar{z}_{12} \text { in } K_{1} \cdot K_{2},
$$

wo $\alpha$ und $\beta$ eindeutig bestimmte Zahlen sind. Wir stellen uns nun zunächst die Aufgabe, aus (17) die Homologien, die $Z_{12}$ in $K_{1}$ oder $K_{2}$ erfüllt, zu 
bestimmen. Wir lösen diese Aufgabe zuerst für einen speziellen Zyklus $Z_{12}^{*}$, der in der ersten Projektion als das Feld der äußeren Normalvektoren $\operatorname{der} S^{n-2}$ definiert ist. Für diesen Zyklus heißt (17):

$$
Z_{12}^{*} \sim z_{12}+\bar{z}_{12} \text { in } K_{1} \cdot K_{2} .
$$

Beweis: $Z_{12}^{*}$ erfüllt eine Homologie

$$
Z_{12}^{*} \sim \alpha^{*} z_{12}+\beta^{*} \bar{z}_{12} \text { in } K_{1} \cdot K_{2} .
$$

Die Bestimmung der Unbekannten $\alpha^{*}$ und $\beta^{*}$ gelingt auf die folgende Weise: Man ordne einem Punkt $p_{1} \times v_{1}$ von $K_{1} \cdot K_{2}$ (siehe (2)) den Punkt $v_{1}$ der $V_{1}^{\prime}=S_{1}^{\prime n-2} \mathrm{zu}$; diese stetige Abbildung $f$ von $K_{1} \cdot K_{2}$ in die $S_{1}^{\prime n-2}$ vermittelt eine homomorphe Abbildung der Betti'schen Gruppen von $K_{1} \cdot K_{2}$ in die Betti'schen Gruppen der $S_{1}^{\prime n-2}$, die $\left(17^{* *}\right)$ in die Homologie $f\left(Z_{12}^{*}\right) \sim \alpha^{*} \cdot f\left(z_{12}\right)+\beta^{*} \cdot f\left(\bar{z}_{12}\right)=\alpha^{*} \cdot S_{1}^{\prime n-2}$ transformiert. Aus $f\left(Z_{12}^{*}\right)=$ $S_{1}^{\prime n-2}$ ergibt sich $\alpha^{*}=1$; auf analoge Weise findet $\operatorname{man} \beta^{*}=1$.

Bezüglich $K_{1}$ erfüllt $Z_{12}^{*}$ die Homologie:

$$
Z_{12}^{*} \sim z_{12} \text { in } K_{1} .
$$

Beweis durch stetige Abänderung von $Z_{12}^{*}$ : Man lasse einen beliebigen Punkt $p_{1} \times v_{1}$ von $Z_{12}^{*}$ den durch das folgende Schema angedeuteten Weg durchlaufen:

$$
p_{1} \times v_{1}, \quad p_{1}(t) \times v_{1}, \quad s \times v_{1} .
$$

Dabei ist $t$ ein von 0 bis 1 laufender Deformationsparameter; $p_{1}(t)$ wandert geradlinig und gleichförmig von $p_{1}$ zum Punkte $s$ der $S^{n-2}$.

Durch Ausübung der Transformation vermittelst reziproker Radien an der $S^{n-2}$ wird aus der Figur 1 die Figur 2, in der man auf analoge Weise findet:

$$
Z_{12}^{*} \sim(-1)^{n} \cdot z_{12} \text { in } K_{2} .
$$

Für den beliebigen Zyklus $Z_{12}$ haben wir nun aus (17) und (17*) $Z_{12} \sim$ $(\alpha-\beta) \cdot z_{12}+\beta \cdot Z_{12}^{*}$ in $K_{1} \cdot K_{2}$, also auch in $K_{1}$; aus $\left(18^{*}\right)$ folgt somit $Z_{12} \sim \alpha \cdot z_{12}$ in $K_{1}$. Analog unter Verwendung von $\left(19^{*}\right): Z_{12} \sim$ $\left[\alpha-\beta+(-1)^{n} \cdot \beta\right] \cdot z_{12}$ in $K_{2}$. Es hat sich also die folgende Lösung unserer Aufgabe ergeben:

$$
\begin{aligned}
& \text { Aus } Z_{12} \sim \alpha z_{12}+\beta \bar{z}_{12} \text { in } K_{1} \cdot K_{2} \\
& \text { folgt } Z_{12} \sim \alpha z_{12} \text { in } K_{1} \\
& \text { und } Z_{12} \sim\left[\alpha-\beta+(-1)^{n} \beta\right] z_{12} \text { in } K_{2} .
\end{aligned}
$$


Aus diesen Formeln ziehen wir nun Folgerungen:

Satz 4. Die $(n-2)$-dimensionale Betti'sche Gruppe von $V_{n, 2}$ ist zyklisch und besitzt für gerades $n$ die Ordnung 0 , für ungerades $n$ die Ordnung 2.

Dabei verstehen wir unter einer zyklischen Gruppe der Ordnung 0 eine freie zyklische Gruppe.

Beweis: Wegen ( 7$)$ bildet unser Zyklus $z_{12}$ eine $(n-2)$-dimensionale Homologiebasis in $K_{1}$; da aber $K_{1}$ und $K_{2}$ durch unsere Transformation vermittelst reziproker Radien topologisch aufeinander abgebildet sind, ist $z_{12}$ auch eine Homologiebasis für $K_{2}$. Nun läßt sich ferner wegen (12) [die Voraussetzung $\left(J_{1}\right)$ ist für $r=n-3$ erfüllt] jeder $(n-2)$-dimensionale Zyklus von $V_{n, 2}$ als Summe eines Zyklus aus $K_{1}$ und eines Zyklus aus $K_{2}$ schreiben. Aus diesen drei Tatsachen folgt, daß die Homologieklasse von $z_{12}$ in $V_{n, 2}$ die Gruppe $B^{n-2}\left(V_{n, 2}\right)$ erzeugt, diese Gruppe ist also zyklisch; um ihre Ordnung festzustellen, müssen wir die Ordnung von $z_{12}$ bestimmen. Sei also etwa $\gamma \cdot z_{12} \sim 0$ in $V_{n, 2}$, d. h. $\gamma \cdot z_{12}=\dot{C}$. Eine Zerlegung $C=C_{1}+C_{2}$ von $C$ liefert $\gamma \cdot z_{12}=\dot{C}_{1}+\dot{C}_{2}$. Dies ist nur möglich für $\dot{C}_{1}=Z_{12}$ und $\dot{C}_{2}=\bar{Z}_{12}$. Wir finden also zunächst:

$$
\gamma \cdot z_{12}=Z_{12}+\bar{Z}_{12} \text { mit } Z_{12} \sim 0 \text { in } K_{1} \text { und } \bar{Z}_{12} \sim 0 \text { in } K_{2} .
$$

Nehmen wir etwa $n$ als ungerade an, so folgt aus $Z_{12} \sim 0$ in $K_{1}$ unter Vermittlung von (18) $Z_{12} \sim \beta \cdot \bar{z}_{12}$ in $K_{1} \cdot K_{2}$. Analog unter Verwendung von (19) $\bar{Z}_{12} \sim 2 \bar{\beta} \cdot z_{12}+\bar{\beta} \cdot \bar{z}_{12}$ in $K_{1} \cdot K_{2}$. Durch Einsetzen in (20) finden wir die Homologie $\gamma \cdot z_{12} \sim 2 \bar{\beta} \cdot z_{12}+(\beta+\bar{\beta}) \cdot \bar{z}_{12}$ in $K_{1} \cdot K_{2}$. Diese Homologie ist nur für $\gamma=2 \cdot \bar{\beta}$ möglich; aus $\gamma z_{12} \sim 0$ in $V_{n, 2}$ folgt also, daß $\gamma$ gerade ist. Die Ordnung von $z_{12}$ ist somit mindestens 2; daß sie genau 2 ist, folgt aus der Betrachtung von $-\bar{z}_{12}$. Nach (18) ist nämlich $-\bar{z}_{12} \sim 0$ in $V_{n, 2}$, und nach (19) $-\bar{z}_{12} \sim 2 \cdot z_{12}$ in $V_{n, 2}$; man hat daher in der Tat $2 z_{12} \sim 0$ in $V_{n, 2}$. Da der Fall eines geraden $n$ analog untersucht werden kann, ist Satz 4 bewiesen.

Zugleich hat sich herausgestellt, daß $z_{12}$ Basiszyklus für die Gruppe $B^{n-2}\left(V_{n, 2}\right)$ ist. Dies ist später von Wichtigkeit; wir geben daher noch eine Definition von $z_{12}$, die unabhängig von unserer Zerlegung der $V_{n, 2}$ ist. Zu diesem Zwecke betrachte man alle 2-Systeme $\sigma_{n, 2}$ der $V_{n, 2}$ (Nr. 1), die im ersten Vektor übereinstimmen. Die Endpunkte der zweiten Vektoren dieser Systeme durchlaufen eine $(n-2)$-dimensionale Sphäre, die wir uns orientiert denken. Dann bilden die Systeme $\sigma_{n, 2}$ einen 
( $n$ - 2)-dimensionalen Zyklus, den wir $z_{n, 2}$ nennen. Es ist leicht ersichtlich, daß $z_{n, 2}$ mit $z_{12}$ identifiziert werden kann; wir finden daher den folgenden

Hilfssatz: Der Zyklus $z_{n, 2}$ ist Basiselement für die $(n-2)$-dimensionale Betti'sche Gruppe der $V_{n, 2}$.

Die Mannigfaltigkeit $V_{n, 2}$ ist orientierbar. Wir werden dies später beweisen. Aus Satz 2 und Satz 4 kann man daher mit Hilfe des Poincare'schen Dualitätssatzes alle Betti'schen Gruppen der $V_{n, 2}$ bestimmen. Man erhält das folgende Resultat:

Satz 5. Für gerades $n$ sind die von 0 verschiedenen Betti'schen Zahlen der $V_{n, 2}: p^{0}=p^{n-2}=p^{n-1}=p^{2 n-3}=1$; Torsion ist nicht vorhanden. Für ungerades $n$ ist auch $p^{n-2}=p^{n-1}=0$, es tritt aber eine $(n-2)$ dimensionale Torsionsgruppe der Ordnung 2 auf.

Weiterhin gestatten uns die Beziehungen (18) und (19), die Klassen der stetigen Abbildungen einer höchstens (n-2)-dimensionalen Sphäre in die $V_{n, 2} \mathbf{z u}$ bestimmen. Es gilt nämlich:

Satz 6. Zwei stetige Abbildungen einer höchstens $(n-2)$-dimensionalen Sphäre in die $V_{n, 2}$ sind homotop, wenn sie denselben Homologietypus ${ }^{10}$ ) besitzen $\left.{ }^{10 a}\right)$.

Wir schicken dem Beweis einige Hilfsbetrachtungen voraus. Sei etwa $f$ eine gegebene stetige Abbildung der Sphäre $S_{0}^{r}(r \leqq n-2)$ in die $V_{n, 2}$ und sei $v_{0}$ ein beliebiger Punkt der $S_{0}^{r}$. Denken wir uns wie in Nr. 1 die $V_{n, 2}$ als Menge aller an die $S^{n-1}$ tangentialen Vektoren des $R^{n}$, so können wir zunächst für alle Homotopieuntersuchungen voraussetzen; daß die Bildvektoren der Punkte $v_{0}$ nicht im Nordpol der $S^{n-1}$ angreifen. (Ist dies noch nicht der Fall, so kann man es wegen $r<n-1$ immer durch eine stetige Abänderung von $f$ erreichen.) Dann gehen also beim Übergang zu unserer ersten Projektion keine Bildvektoren verloren, und man hat nach (2) $f\left(v_{0}\right)=p_{1} \times v_{1}$. Weiterhin kann man sogar voraussetzen, daß nur Punkte $s \times v_{1}$ (siehe Fig. 1) als Bildpunkte $f\left(v_{0}\right)$ auftreten. (In der Tat kann die stetige Abbildung $v_{0} \rightarrow p_{1} \times v_{1}$ durch den Deformationsprozeß $(D)$ (Anfang dieser Nr.) stetig in eine Abbildung abgeändert werden, die die gewünschte Eigenschaft hat.) Wir setzen also voraus:

$$
f\left(v_{0}\right)=s \times v_{1} .
$$

10) AH: Kap. VIII, § 3.

10a) Dieser Satz ist eine Verallgemeinerung des Satzes über die Klassifikation der Sphërenabbildungen (AH, Kap. XIII, § 2). 
Die Abbildung $\varphi\left(v_{0}\right)=v_{1}$ der $S_{0}^{r}$ in die der $V_{n, 2}$ zugeordnete Mannigfaltigkeit $S_{1}^{\prime n-2}$ nennen wir die der Abbildung $f$ zugeordnete Abbildung $\varphi$. Ist nun $\bar{f}$ eine zweite Abbildung der $S_{0}^{r}$ in die $V_{n, 2}$ und $\bar{\varphi}$ ihre zugeordnete Abbildung, so gilt:

Aus der Homotopie von $\varphi$ und $\bar{\varphi}$ folgt die Homotopie von $f$ und $\bar{f}$.

Dies folgt einfach daraus, daß die Multiplikation mit dem festen Punkt $s$ eine topologische Abbildung der $S_{1}^{\prime n-2}$ in die $V_{n, 2}$ ist.

Nun gehen wir zum Beweis von Satz 6 über. Es sind drei Fälle zu unterscheiden:

Fall 1. $r<n-2$. Nach Satz 2 müssen wir zeigen, daß jede Abbildung der $S_{0}^{r}$ in die $V_{n, 2}$ 0-homotop ist, daß also das Bild der $S_{0}^{r}$ auf einen Punkt zusammengezogen werden kann. Nach (22) ist dies aber eine Folge der Tatsache, daß wegen $r<n-2$ die zugeordnete Abbildung 0-homotop ist.

Fall 2. $r=n-2$ und $n$ ist gerade. $f$ und $\bar{f}$ seien die beiden Abbildungen, von denen in Satz 6 die Rede ist. Verstehen wir unter $S_{0}^{r}=S_{0}^{n-2}$ auch den Zyklus, den diese Sphäre in einer fest gewählten Orientierung darstellt, so besagt die Voraussetzung von Satz $6 f\left(S_{0}^{n-2}\right) \sim \bar{f}\left(S_{0}^{n-2}\right)$ in $V_{n, 2}=K_{1}+K_{2}$. Wegen (21) sind $f\left(S_{0}^{n-2}\right)$ und $\bar{f}\left(S_{0}^{n-2}\right)$ Zyklen in $K_{1} \cdot K_{2}$, erfüllen also Homologien (17): $f\left(S_{0}^{n-2}\right) \sim \alpha z_{12}, \bar{f}\left(S_{0}^{n-2}\right) \sim \bar{\alpha} z_{12}$ in $K_{1} \cdot K_{2}$; man hat demnach $\alpha \cdot z_{12} \sim \bar{\alpha} \cdot z_{12}$ in $V_{n, 2}$. Dies ist nach Satz 4 nur für $\alpha=\bar{\alpha}$ möglich, und es ergibt sich endlich $f\left(S_{0}^{n-2}\right) \sim \bar{f}\left(S_{0}^{n-2}\right)$ in $K_{1} \cdot K_{2}$. Diese Homologie bilden wir in die $S_{1}^{\prime n-2}$ ab, indem wir dem Punkt $p_{1} \times v_{1}$ von $K_{1} \cdot K_{2}$ den Punkt $v_{1}$ zuordnen. So findet $\operatorname{man} \varphi\left(S_{0}^{n-2}\right) \sim \bar{\varphi}\left(S_{0}^{n-2}\right)$ in $S_{1}^{\prime n-2}$. Die beiden Abbildungen $\varphi$ und $\bar{\varphi}$ der $S_{0}^{n-2}$ in die $S_{1}^{\prime n-2}$ haben also denselben Abbildungsgrad, woraus ihre Homotopie folgt. (22) beendet den Beweis.

Fall 3. $r=n-2$ und $n$ ungerade. Dann ergibt sich aus Satz 4 nur $\alpha \equiv \bar{\alpha}(\bmod 2)$. Sei etwa $\bar{\alpha}=\alpha-2 k$. Der obige Beweis funktioniert nun auch in diesem Fall, wenn̄ wir zeigen können, daß unsere Abbildung $f$ mit $f\left(S_{0}^{n-2}\right) \sim \alpha z_{12}$ in $K_{1} \cdot K_{2}$ stetig in eine der Bedingung (21) genügende Abbildung $f_{2}$ mit $f_{2}\left(S_{0}^{n-2}\right) \sim(\alpha-2 k) z_{12}$ in $K_{1} \cdot K_{2}$ abgeändert werden kann. Zu diesem Zweck sei $F$ eine beliebige Abbildung der $S_{0}^{n-2}$ in die $S^{n-2}$ vom Grade $k$. Zunächst wird nun $f$ nach dem folgenden Schema in eine Abbildung $f_{1}$ abgeändert:

$$
f\left(v_{0}\right)=s \times v_{1}, F\left(v_{0}, 1-t\right) \times v_{1}, F\left(v_{0}\right) \times v_{1}=f_{1}\left(v_{0}\right) .
$$

$F\left(v_{0}, t\right)$ läuft wieder geradlinig und gleichförmig von $F\left(v_{0}\right)$ nach $s$. Der Zyklus $f_{1}\left(S_{0}^{n-2}\right)$ liegt wieder in $K_{1} \cdot K_{2}$ und erfüllt dort die Homologie 
$f_{1}\left(S_{0}^{n-2}\right) \sim \alpha z_{12}+k \bar{z}_{12}$, was man analog wie $\left(17^{*}\right)$ beweist. Nach (19) ist $f_{1}\left(S_{0}^{n-2}\right) \sim(\alpha-2 k) z_{12}$ in $K_{2}$. Nun geht man durch die Transformation vermittelst reziproker Radien in die Figur 2 und ändert dort $f_{1}$ nach dem zu $(D)$ analogen Deformationsproze $B$ ab. Das Resultat ist eine der Bedingung (21) genügende Abbildung $f_{2}$ mit $f_{2}\left(S_{0}^{n-2}\right) \sim(\alpha-2 k) z_{12}$ in $K_{2}$ und $f_{2}\left(S_{0}^{n-2}\right) \sim \delta z_{12}$ in $K_{1} \cdot K_{2}$. Für die Unbekannte $\delta$ findet man aus (19) leicht $\delta=\alpha-2 k$. Damit ist Satz 6 vollständig bewiesen.

Aus Satz 2 und Satz 6 folgt noch

Satz \%. Für $n>3$ ist die Mannigfaltigkeit $V_{n, 2}$ einfach zusammenhängend, also auch orientierbar.

Die Mannigfaltigkeit $V_{3,2}$ nimmt also als nicht einfach zusammenhängende Mannigfaltigkeit unter den $V_{n, 2}$ eine Sonderstellung ein, die wir später $(\S 5, \mathrm{Nr} .3)$ bei der Untersuchung der Parallelisierbarkeit dreidimensionaler Mannigfaltigkeiten ausnützen werden. Wir erwähnen noch, daß $V_{3,2}$ zum dreidimensionalen projektiven Raum $P^{3}$ homöomorph ist. Zum Beweise beachte man, da $V_{3,2}$ als Menge der Linienelemente einer zweidimensionalen Sphäre zur Gruppe der euklidischen Drehungen dieser Sphäre homöomorph ist. Eine derartige Drehung ist aber durch vier homogene Parameter eindeutig bestimmt.

\section{Topologie der $V_{n, m}$.}

Die Vereinigung der Resultate der Abschnitte 2 und 3 erlaubt die Herleitung weiterer topologischer Eigenschaften der $V_{n, m}$. Durch Induktion in der Reihe (4) der zugeordneten Mannigfaltigkeiten, die aber jetzt bei der Mannigfaltigkeit $V_{n-m+2,2}$ abgebrochen wird, beweist man die folgenden Sätze, bei denen immer $m>1$ vorausgesetzt ist:

1. Die Betti'sche Gruppe $B^{n-m}\left(V_{n, m}\right)$ ist zyklisch von der Ordnung 0 für gerades $n-m$ und von der Ordnung 2 für ungerades $n-m$.

Beweis aus Satz 3 und Satz 4. Um einen Basiszyklus für $B^{n-m}\left(V_{n, m}\right)$ zu finden, betrachte man alle $m$-Systeme $\sigma_{n, m}$ der $V_{n, m}$ (Nr. 1), deren $(m-1)$ erste Vektoren fest vorgegeben sind. Die Endpunkte der letzten Vektoren dieser Systeme durchlaufen eine $(n-m)$-dimensionale Sphäre, die wir uns orientiert denken. Die Systeme $\sigma_{n, m}$ bilden dann einen $(n-m)$-dimensionalen Zyklus $z_{n, m}$.

2. $z_{n, m}$ ist Basiszyklus für $B^{n-m}\left(V_{n, m}\right)$.

Beweis aus den beiden Hilfssätzen in Nr. 2 und Nr. 3. 
3. Zwei stetige Abbildungen einer höchstens $(n-m)$-dimensionalen Sphäre in die $V_{n, m}$ sind homotop, wenn sie denselben Homologietypus besitzen.

Sind $f$ und $\bar{f}$ die beiden Abbildungen, so definiere man zum Beweise analog wie in Nr. 3 die zugeordneten Abbildungen $\varphi$ und $\bar{\varphi}$ in die $V_{n-1, m-1}$. Aus der Homotopie der zugeordneten Abbildungen folgt dann die Homotopie von $f$ und $\bar{f}$.

Wegen 3. ist $V_{n, m}$ für $m<n-1$ einfach zusammenhängend, also auch orientierbar. $V_{n, n-1}$ ist zur Gruppe der euklidischen Drehungen einer $(n-1)$-dimensionalen Sphäre homöomorph und ist somit als Gruppenmannigfaltigkeit orientierbar. Für diese Mannigfaltigkeit gilt übrigens

4. Die Fundamentalgruppe der $V_{n, n-1}$ ist eine zyklische Gruppe der Ordnung $2(n>2)$.

Der Beweis ist nur unwesentlich vom Beweis von 3. verschieden. (Zur Verankerung der Induktion beachte man, daß 4 . für $V_{3,2}$ aus der Homöomorphie zum projektiven Raum folgt.)

Zum Schluß wollen wir aus diesen Sätzen noch die Eigenschaften der $V_{n, m}$ herleiten, die wir für das folgende brauchen:

Satz 8. Das stetige Bild einer höchstens $(n-m-1)$-dimensionalen Sphäre in der $V_{n, m}$ ( $m$ beliebig) ist auf einen Punkt zusammenziehbar.

Beweis aus 3 . und Satz 2.

Satz 9. Ist f eine stetige Abbildung einer orientierten Sphäre $S_{0}^{n-m}$ in die $V_{n, m}$, so gilt die Homologie:

$$
f\left(S_{0}^{n-m}\right) \sim \alpha z_{n, m} \quad \text { in } V_{n, m} .
$$

Ist $n-m$ gerade oder $m=1$, so ist a eindeutig bestimmt, und zwei $A b$ bildungen mit demselben Wert von a sind homotop.

Ist aber $n-m$ ungerade und $m$ von 1 verschieden, so ist a nur (mod 2) bestimmt $\left.{ }^{11}\right)$; zwei Abbildungen, zu denen (mod 2) kongruente Werte von $\alpha$ gehören, sind homotop.

\section{§ 2. Die offenen Mannigfaltigkeiten $V_{n, m}^{*}$}

\section{Definitionen}

In diesem Abschnitt wollen wir uns von der Beschränkung auf orthogonale und normierte $m$-Systeme frei machen. Wir definieren: Ein ge-

11) daher können wir im folgenden voraussetzen, $\operatorname{da} B a$ in diesem Fall entweder den Wert 0 oder 1 hat. 
ordnetes System $\sigma_{n, m}{ }^{*}$ von $m$ linear unabhängigen Vektoren $\mathfrak{v}_{1}, \mathfrak{v}_{2}, \ldots$, $\mathfrak{y}_{m}$, die in einem Punkte des $R^{n}$ angreifen, heißt ein affines $m$-System des $R^{n}$. Die Systeme $\sigma_{n, m}$ von $\S 1$ nennen wir jetzt orthogonale $m$ Systeme, um sie von den affinen $m$-Systemen zu unterscheiden. $m$ erfülle wieder die Ungleichungen

$$
0<m<n .
$$

Die Menge aller in einem festen Punkt des $R^{n}$ angreifenden affinen $m$-Systeme heißt $V_{n, m}^{*}$. Ein System $\sigma_{n, m}^{*}$ ist durch die $n \cdot m$ Komponenten seiner Vektoren gegeben, kann also als Punkt eines $(n \cdot m)$ dimensionalen Zahlenraumes aufgefaßt werden. In dieser Auffassung wird $V_{n . m}^{*}$ zu einem Teilgebiet des Zahlenraumes, ist also eine offene Mannigfaltigkeit.

\section{Retrahierende Abbildung}

Wir ersetzen in jedem $m$-System der $V_{n, m}^{*}$ den Vektor $\mathfrak{y}_{i}$ durch den Vektor

$$
\mathfrak{v}_{i}^{\prime}=\mathfrak{y}_{i}-\left(\mathfrak{v}_{i} \cdot \mathfrak{v}_{j}\right) \mathfrak{y}_{j}
$$

$i$ und $j$ sind fest gewählt, aber voneinander verschieden; $\left(\mathfrak{v}_{i} \cdot \mathfrak{v}_{j}\right)$ bedeutet das skalare Produkt von $\mathfrak{y}_{i}$ und $\mathfrak{y}_{j}$. Es entsteht eine stetige Abbildung $f$ von $V_{n, m}^{*}$ in sich; die Bildmenge bezeichnen wir mit $f\left(V_{n, m}^{*}\right)$. Durch Betrachtung der Abbildungsschar

$$
\mathfrak{v}_{i}^{\prime}(t)=\mathfrak{v}_{i}-t\left(\mathfrak{v}_{i} \cdot \mathfrak{v}_{j}\right) \mathfrak{v}_{j}, \quad(0 \leqq t \leqq 1)
$$

erkennt man, daß $f$ eine Deformation ist, $d . h$. zur Klasse der Identität gehört. Ersetzt man für einen bestimmten Wert von $k$ in jedem System $\sigma_{n, m}^{*} \operatorname{der} V_{n, m}^{*}$ den Vektor $\mathfrak{y}_{k}$ durch den Vektor

$$
\mathfrak{v}_{k}^{\prime}=\frac{\mathfrak{v}_{k}}{\left|\mathfrak{v}_{k}\right|},
$$

so ist damit eine andere stetige Abbildung $g$ von $V_{n, m}^{*}$ in sich gegeben. Auch $g$ ist eine Deformation, wie sich aus der Abbildungsschar

$$
\mathfrak{v}_{k}^{\prime}(t)=\left[t+(1-t) \cdot\left|\mathfrak{v}_{k}\right|\right] \frac{\mathfrak{v}_{k}}{\left|\mathfrak{w}_{k}\right|} \quad(0 \leqq t \leqq 1)
$$

ergibt. Die beiden Abbildungen $f$ und $g$ lassen die Mannigfaltigkeit $V_{n, m}$, die ja Teilmenge von $V_{n, m}^{*}$ ist, invariant. 
Mit $f\left(V_{n, m}^{*}\right)$ oder $g\left(V_{n, m}^{*}\right)$ kann man wieder eine Deformation vom Typus (2) bezw. (3) vornehmen und schließlich durch Zusammensetzen von endlich vielen Deformationen dieser Art eine Deformation $F$ konstruieren, die $V_{n, m}^{*}$ stetig auf $V_{n, m}$ abbildet. Dies folgt aus der von der analytischen Geometrie her bekannten Tatsache, da $\beta$ sich jedes affine System $\sigma_{n, m}^{*}$ der $V_{n, m}^{*}$ durch endlich viele Schritte vom Typus (2) und (3) orthogonalisieren läßt. Die Deformation $F$ nennen wir die retrahierende Abbildung ${ }^{12}$ ) von $V_{n, m}^{*}$ auf $V_{n, m}$.

\section{Topologie der $V_{n, m}^{*}$}

Mit Hilfe unserer Retraktion können wir nun die Ergebnisse von $\S 1$, Nr. 4, auf die offene Mannigfaltigkeit $V_{n, m}^{*}$ übertragen:

Satz 10. $V_{n, m}^{*}$ ist zu $V_{n, m}$ vollständig homologie-äquivalent, d. h. es gilt für beliebiges $r: B^{r}\left(V_{n, m}^{*}\right) \approx B^{r}\left(V_{n, m}\right)$; ferner gelten alle in $\S 1, N r .4$ für die $V_{n, m}$ bewiesenen Resultate auch für die Mannigfaltigkeit $V_{n, m}^{*}$.

Beweis: Die retrahierende Abbildung $F$ induziert eine homomorphe Abbildung von $B^{r}\left(V_{n, m}^{*}\right)$ in $B^{r}\left(V_{n, m}\right)$. Um nachzuweisen, daß dieser Homomorphismus ein Isomorphismus ist, genügt es (da jede $r$-dimensionale Homologieklasse von $V_{n, m}$ trivialerweise als Bildklasse auftritt) zu zeigen, daß sein Kern aus der Nullklasse allein besteht. Sei also etwa $z^{r}$ ein Zyklus in $V_{n, m}^{*}$ und $F\left(z^{r}\right) \sim 0$ in $V_{n, m}$, also auch in $V_{n, m}^{*}$. Da $F\left(z^{r}\right)$ durch Deformation aus $z^{r}$ hervorgegangen ist, hat man $F\left(z^{r}\right) \sim z^{r}$ in $V_{n, m}^{*}$, also tatsächlich $z^{r} \sim 0$ in $V_{n, m}^{*}$.

Die zweite Behauptung von Satz 10 läßt sich nun mit Hilfe unserer Retraktion leicht beweisen.

Bemerkung: Alle positiv orientierten und in einem festen Punkte des $R^{n}$ angreifenden $n$-Systeme bilden eine Mannigfaltigkeit, die zur Gruppe $A_{n}$ aller eigentlichen affinen Abbildungen des $R^{n}$ homöomorph ist. Aus unseren Untersuchungen folgt leicht, daß $A_{n}$ zur $V_{n, n-1}$ vollständig homologie-äquivalent ist, und daß die Fundamentalgruppe von $A_{n}$ für $n>2$ eine zyklische Gruppe der Ordnung 2 ist.

\section{§ 3. Vektorfelder im euklidischen Raum, Charakteristik}

\section{Charakteristik eines $m$-Feldes auf einer Sphäre}

In diesem Abschnitt verstehen wir unter $E^{r+1}$ eine in den euklidischen $n$-dimensionalen Raum $R^{n}$ eingebettete $(r+1)$-dimensionale krumme

12) Dieser Begriff stammt von $K$. Borsuk; vgl. AH: Kap. VIII, $\$ 6$. 
Zelle und unter $S^{r}$ die Randsphäre von $E^{r+1}$. Bezeichnen wir einen Punkt der $S^{r}$ mit $p$, so können wir die Punkte der Zelle $E^{r+1}$ durch ein Polarkoordinatensystem $\varrho, p$ festlegen. ( $\varrho$ ist eine von 0 bis 1 laufende Zahl, der Punkt $(0, p)$ ist der Ursprung des Koordinatensystems, $(1, p)$ ist mit $p$ identisch).

Ist in jedem Punkte von $S^{r}$ ein affines $m$-System $\sigma(p)$ des $R^{n}$ angebracht, so sprechen wir von einem $m$-Feld $\mathfrak{F}$ auf $S^{r}$. Die Untersuchung dieser Felder ist das Ziel des Paragraphen. Zu diesem Zweck wählen wir eine in den $R^{n}$ eingebettete Vektormenge $V_{n, m}^{*}$, und ordnen dem Punkt $p$ von $S^{r}$ das zu $\sigma(p)$ parallele $m$-System der $V_{n, m}^{*}$ zu. Durch diese $\mathrm{Zu}$ ordnung ist eine Abbildung $f$ der Sphäre $S^{r}$ in die Mannigfaltigkeit $V_{n, m}^{*}$ gegeben, die wir Abbildung durch parallele $m$-Systeme nennen. Wir nennen ferner das Feld $\mathfrak{F}$ stetig, falls $f$ stetig ist; dies wird im folgenden immer vorausgesetzt. In analoger Weise definieren wir ein stetiges Feld in der Zelle $E^{r+1}$ und die zugehörige Abbildung durch parallele $m$-Systeme.

Nun stellt sich sofort die Frage, unter welchen Bedingungen ein auf $S^{r}$ gegebenes stetiges Feld $\sigma(p)$ zu einem in $E^{r+1}$ stetigen Feld $\sigma(\varrho, p)$ erweitert werden kann; $[\sigma(1, p)=\sigma(p)]$. Ist die Dimension $r$ unserer Sphäre kleiner als $n-m$, so zeigt Satz $8(10)$, daß diese Fortsetzung immer möglich ist. In der Tat ist dann $f\left(S^{r}\right)$ in $V_{n, m}^{*} 0$-homotop, also ${ }^{13}$ ) kann $f$ zu einer in $E^{r+1}$ stetigen Abbildung in die $V_{n, m}^{*}$ ergänzt werden. Ist aber $r=n-m$, also die Sphäre $(n-m)$-dimensional (und orientiert), so folgt aus Satz 9 (10), daß die verlangte Fortsetzung dann und nur dann möglich ist, wenn die zu unserer Abbildung $f$ durch parallele $m$-Systeme gehörige Zahl $\alpha$ verschwindet.

Die Zahl $\alpha$ heißt die Charakteristik des $m$-Feldes $\mathscr{F}$ auf der Sphäre $S^{r}=S^{n-m}$. Man findet also:

Satz 11. Ein auf der Randsphäre einer Zelle gegebenes stetiges $m$-Feld kann stetig ins Innere fortgesetzt werden:

a) wenn die Dimension der Sphäre kleiner als $n-m$ ist,

b) wenn die Sphäre $(n-m)$-dimensional und die Charakteristik des Feldes auf ihr 0 ist.

Fortsetzung durch Zentralprojektion:

Durch die Definition: , , $\sigma(\varrho, p)$ parallel zu $\sigma(p)^{\text {“ }}$ kann ein Randfeld immer ins Innere der Zelle $E^{r+1}$ fortgesetzt werden. Diese Fortsetzung

13) AH: Kap. XIII, § 1, Hilfssatz II. 
nennen wir Fortsetzung durch Zentralprojektion vom Punkte $(0, p)$ aus. Dabei wird aber im allgemeinen die Stetigkeit des fortgesetzten Feldes im Projektionszentrum durchbrochen sein. Ist ferner auf der Randphäre $S^{r}$ ein beliebiges nicht notwendigerweise stetiges $m$-Feld gegeben, und bezeichnen wir die Menge seiner Unstetigkeitsstellen mit $M$, so ist das durch Zentralprojektion in die Zelle $E^{r+1}$ fortgesetzte Feld in allen Punkten des Kegels über $M$ mit dem Projektionszentrum als Spitze unstetig.

\section{Bemerkungen zur Berechnung der Charakteristik}

Es erweist sich in manchen Fällen als nützlich, die Charakteristik auf andere Weise als vermittelst der Abbildung durch parallele $m$-Systeme zu berechnen: Es sei in der Zelle $E^{n-m+1}$ ein stetiges Feld $\mathfrak{B}$ von positiv orientierten $n$-Systemen $\beta(\varrho, p)$ gegeben. Ein derartiges Feld heißt Basisfeld in der Zelle $E^{n-m+1}$. (Positiv orientiert heißt gleich orientiert wie das System $\mathfrak{k}_{1}, \mathfrak{e}_{2}, \ldots, \mathfrak{e}_{n}$ der Grundvektoren des $R^{n}$ ). Um nun die Charakteristik eines auf der $S^{n-m}$ gegebenen $m$-Feldes $\sigma(p)$ zu berechnen, gehen wir folgendermaßen vor: $\mathfrak{v}_{\mu}(\mu=1,2, \ldots, m)$ sei ein Vektor von $\sigma(p)$ und $v_{\mu i}(i=1,2, \ldots, n)$ seine Komponenten bezüglich der Basis $\beta(1, p)$. Ordnet man nun jedem Vektor $\mathfrak{v}_{\mu}$ denjenigen Vektor zu, der im 0 -Punkt des $R^{n}$ angreift und der bezüglich $\mathfrak{e}_{1}, \mathfrak{e}_{2}, \ldots, \mathfrak{e}_{n}$ die Komponenten $v_{\mu i}$ hat, so entsteht eine stetige Abbildung $f^{\prime} \operatorname{der} S^{n-m}$ in die im 0-Punkt des $R^{n}$ angebrachte $V_{n, m}^{*}$. Zu dieser Abbildung gehört nach Satz 9 (10) eine Zahl $\alpha^{\prime}$; wir beweisen, daß $\alpha^{\prime}$ die Charakteristik des gegebenen $m$-Feldes auf $S^{n-m}$ ist.

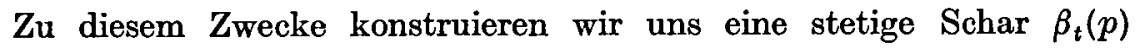
$(0 \leqq t \leqq 1)$ von Basisfeldern auf $\operatorname{der} S^{n-m}$, so daß $\beta_{0}(p)=\beta(1, p)$ und $\beta_{1}(p)$ zu $\mathfrak{e}_{1}, \mathfrak{e}_{2}, \ldots, \mathfrak{e}_{n}$ parallel ist. (Zur Konstruktion dieser Schar definiere man etwa für $0 \leqq t \leqq \frac{1}{2}: \beta_{t}(p)$ parallel zu $\beta(1-2 t ; p)$; die Systeme $\beta_{1 / 2}(p)$ sind dann untereinander parallel und können leicht durch

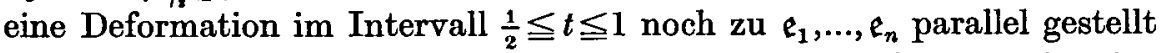
werden.) $\mathrm{Zu}$ jedem Basisfeld $\beta_{t}(p)$ gehört eine stetige und sich stetig mit $t$ ändernde Abbildung $f_{t}^{\prime}$ der $S^{n-m}$ in die $V_{n, m}^{*}$. $f_{0}^{\prime}$ ist unser $f^{\prime}$, während $f_{1}^{\prime}$ mit der Abbildung $f$ durch parallele $m$-Systeme identisch ist. $f$ und $f^{\prime}$ sind somit homotop; daraus folgt die Behauptung.

Berechnung der Charakteristik durch Rekursion:

Unsere neue Methode zur Berechnung der Charakteristik ist sehr nützlich, wenn die folgende Situation vorliegt: 
a) Die Zelle $E^{n-m+1}$ liege in einer $n^{\prime}$-dimensionalen Ebene $R^{n^{\prime}}$ des $R^{n}$. $\left(n^{\prime}<n\right) . R^{n^{\prime}}$ werde etwa durch die Grundvektoren $\mathfrak{e}_{n-n^{\prime}+1}, \ldots, \mathfrak{e}_{n}$ aufgespannt.

b) Die Vektoren $\mathfrak{v}_{1}, \mathfrak{y}_{2}, \ldots, \mathfrak{v}_{n-n}$, des Systems $\sigma(p)$ seien nicht im $R^{n^{\prime}}$ enthalten; sie bilden dann ein $\left(n-n^{\prime}\right)$-System des $R^{n}$, und alle diese Systeme bilden ein $\left(n-n^{\prime}\right)$-Feld auf $S^{n-m}$. Wir setzen voraus, daß dieses Feld zu einem $\left(n-n^{\prime}\right)$-Feld $\overline{\boldsymbol{\sigma}}(\varrho, p)$ in $E^{n-m+1}$ ergänzt werden kann.

c) Die Vektoren $\mathfrak{y}_{n-n^{\prime}+1}, \ldots, \mathfrak{v}_{m}$ von $\sigma(p)$ seien im $R^{n \prime}$ enthalten; sie bilden dann ein $m^{\prime}$-System $\sigma^{\prime}(p)$ des $R^{n}$. $\left(m^{\prime}=m-n+n^{\prime}\right)$.

$\sigma(p)$ und $\sigma^{\prime}(p)$ besitzen Charakteristiken $\alpha$ und $\alpha^{\prime}$ auf $S^{n-m}$. Dann gilt:

$$
\alpha \equiv \alpha^{\prime}(\bmod 2) \text {. }
$$

(Man könnte unter gewissen Orientierungsvoraussetzungen sogar die Gleichheit von $\alpha$ und $\alpha^{\prime}$ beweisen; für unsere weiteren Zwecke genügt aber die Kongruenz mod 2.)

Beweisandeutung: Man wähle im $R^{n \prime}$ in der Zelle $E^{n-m+1}$ ein Basisfeld $\beta^{\prime}(\varrho, p)$. Dieses Basisfeld wird durch $\bar{\sigma}(\varrho, p)$ zu einem Basisfeld $\beta(\varrho, p)$ im $R^{n}$ ergänzt. Man berechne die gesuchten Charakteristiken bezüglich dieser Basisfelder, wobei man zweckmäßig den Basiszyklus $z_{n, m}$ der $V_{n, m}^{*}(\S 1, N r .4)$ von den im 0-Punkt des $R^{n}$ angreifenden orthogonalen $m$-Systemen, deren $(m-1)$ erste Vektoren $\mathfrak{e}_{1}, \mathfrak{e}_{2}, \ldots, \mathfrak{e}_{m-1}$ sind, durchlaufen läßt.

\section{Charakteristik eines Feldpaares auf einer Zelle.}

Sind auf unserer Zelle $E^{r+1}$ zwei stetige $m$-Felder $\sigma_{0}(\varrho, p)$ und $\sigma_{1}(\varrho, p)$ gegeben, und ist ferner auf der Randsphäre $S^{r}$ für $0 \leqq t \leqq 1$ eine stetige Schar $\sigma_{t}(p)$ von $m$-Feldern konstruiert, die den Randbedingungen $\sigma_{0}(p)=\sigma_{0}(1, p)$ und $\sigma_{1}(p)=\sigma_{1}(1, p)$ genügt, so sprechen wir von einem Feldpaar in $E^{r+1}$. Ein Feldpaar besteht also aus zwei Feldern auf einer Zelle, die am Rande durch eine stetige Schar verbunden sind.

Wir wollen nun untersuchen, unter welchen Bedingungen diese stetige Verbindung ins Innere der Zelle fortgesetzt werden kann. Es soll also eine stetige Schar von $m$-Feldern $\sigma_{t}(\varrho, p)$ in $E^{r+1}$ konstruiert werden, die der Forderung $\sigma_{t}(1, p)=\sigma_{t}(p)$ genügt. Diese Untersuchung läßt sich leicht mit Hilfe der Sätze 8 und 9 unter Berücksichtigung des Satzes 10 durchführen, wenn die Dimension $r+1$ der Zelle höchstens $n-m$ ist: 
Es sei etwa $T$ die (orientierte) Einheitsstrecke, die vom Parameter $t$ durchlaufen wird. Dann konstruieren wir in abstracto den Zylinder $Z$, der als topologisches Produkt $T \times E^{r+1}$ definiert wird und bezeichnen seine Punkte mit $t \times(\varrho, p)$. Wir ordnen weiter dem Punkt $0 \times(\varrho, p)$ von $Z$ das im 0-Punkt des $R^{n}$ angreifende und zu $\sigma_{0}(\varrho, p)$ parallele System [analog für $1 \times(\varrho, p)]$ und dem Punkte $t \times(1, p)$ das im 0-Punkt des $R^{n}$ angreifende und zu $\sigma_{t}(p)$ parallele System zu. Damit ist eine stetige Abbildung $f$ des Randes von $Z$ in die $V_{n, m}^{*}$ gegeben. Ist $r+1<n-m$, so kann $f$ zu einer stetigen Abbildung des ganzen Zylinders in die $V_{n, m}^{*}$ fortgesetzt werden, womit auch die Fortsetzung unserer stetigen Verbindung konstruiert ist. Ist aber $r+1=n-m$, so ist die Fortsetzung dann und nur dann möglich, wenn die nach Satz 9 (10) zu $f$ gehörige Zahl $\alpha$, die wir die Charakteristik des Feldpaares auf $E^{r+1}$ nennen, verschwindet. Um diese Charakteristik zu berechnen, muß der Zylinderrand orientiert werden; da $Z$ ein Produkt ist, kann eine derartige Orientierung durch eine Orientierung der Zelle $E^{r+1}$ gegeben werden. Es gilt also:

Satz 12. Die zu einem Feldpaar gehörige verbindende Randschar kann ins Innere fortgesetzt werden:

a) wenn die Dimension der Zelle, auf der das Paar liegt, kleiner als $(n-m)$ ist.

b) wenn diese Dimension $(n-m)$ und die Charakteristik des Feldpaares auf der (orientierten) Zelle 0 ist.

Wir geben noch eine Beziehung zwischen der Charakteristik eines Feldes und eines Feldpaares. Es seien auf der Sphäre $S^{n-m}$ zwei beliebige stetige $m$-Felder $\mathfrak{F}$ und $\mathfrak{F}^{\prime}$ mit den Charakteristiken $\alpha$ und $\alpha^{\prime}$ gegeben. Ferner sei $S^{n-m}$ in die Zellen $E_{i}^{n-m}$ zerlegt, und auf dem Komplex $K$ der $(n-m-1)$-dimensionalen Zellen dieser Zellenzerlegung seien $\mathfrak{F}$ und $\mathfrak{F}^{\prime}$ durch eine stetige Feldschar verbunden. Damit ist auf jeder Zelle $E_{i}^{n-m}$ ein Feldpaar gegeben, dessen Charakteristik wir mit $\alpha_{i}$ bezeichnen. (Die Zellen $E_{i}^{n-m}$ seien kohärent zu der bei der Berechnung von $\alpha$ und $\alpha^{\prime}$ verwendeten Orientierung der $S^{n-m}$ orientiert.) Dann ist:

$$
\begin{aligned}
& \alpha^{\prime}=\alpha+\underset{(i)}{\sum} \alpha_{i} \text { für gerades } n-m \text { oder } m=1 . \\
& \alpha^{\prime} \equiv \alpha+\sum \alpha_{i}(\bmod 2) \text { für ungerades } n-m \text { und } m \neq 1 .
\end{aligned}
$$

Diese Formel bildet das Fundament der folgenden Untersuchungen; sie ist leicht zu beweisen: 
Man konstruiere den orientierten Produktkomplex $T \times S^{n-m}=$ $T \times \Sigma E_{i}^{n-m}=\Sigma Z_{i}$, wo $Z_{i}$ den über $E_{i}^{n-m}$ konstruierten und beim Beweis von Satz 12 verwendeten Zylinder bedeutet. Durch Randbildung entsteht die Beziehung:

$$
\left(1 \times S^{n-m}\right)-\left(0 \times S^{n-m}\right)=\Sigma \dot{Z}_{i} .
$$

Die Zylinder $Z_{i}$ bilden eine Zellenzerlegung von $T \times S^{n-m}$; bildet man jedes $\dot{Z}_{i}$ wie beim Beweis von Satz 12 in die $V_{n, m}^{*}$ ab, so ist damit auf dem Komplex der $(n-m)$-dimensionalen Zellen dieser Zellenzerlegung eine stetige Abbildung $F$ in die $V_{n, m}^{*}$ gegeben, und es folgt aus $(R)$ :

$$
F\left(1 \times S^{n-m}\right)-F\left(0 \times S^{n-m}\right) \sim \Sigma F\left(\dot{Z}_{i}\right) \text { in } V_{n, m}^{*},
$$

und aus den Definitionen von $\alpha, \alpha^{\prime}$ und $\alpha_{i}$ :

$$
\alpha^{\prime} z_{n},{ }_{m}-\alpha z_{n},{ }_{m} \sim \Sigma \alpha_{i} z_{n},{ }_{m} \text { in } V_{n, m}^{*} .
$$

Aus dieser Homologie und aus Satz 9 und Satz 10 folgen die Behauptungen.

Es muß hier noch der folgende Spezialfall eines Feldpaares erwähnt werden: Ein Feldpaar mit $\sigma_{0}(1, p)=\sigma_{t}(p)=\sigma_{1}(1, p)$ nennen wir ein Feldpaar mit starren Randwerten; es besteht aus zwei in der Zelle $E^{r+1}$ gegebenen und am Rande $S^{r}$ übereinstimmenden stetigen $m$-Feldern. (Die verbindende Randschar fällt mit den gemeinsamen Randwerten der beiden Felder zusammen.) Aus Satz 12 folgt nun: Das erste Feld eines auf $E^{n-m}$ gegebenen Feldpaares mit starren Randwerten kann dann und nur dann unter Festhaltung seines Randfeldes auf der $S^{n-m-1}$ in das zweite Feld deformiert werden, wenn die Charakteristik des Paares auf $E^{n-m}$ verschwindet.

\section{Felder und Feldpaare mit vorgegebener Charakteristik}

Für das folgende brauchen wir einen topologischen. Hilfssatz:

Sei $S^{k}$ eine $k$-dimensionale Sphäre, die in die beiden $k$-dimensionalen Zellen $E$ und $E^{\prime}$ zerlegt ist, und sei $P$ ein zusammenhängendes Polyeder. Dann kann eine stetige Abbildung $f_{1}$ von $E$ in das Polyeder $P$ zu einer stetigen Abbildung der $S^{k}$ erweitert werden, die einer vorgegebenen Abbildungsklasse von $S^{k}$ in das Polyeder $P$ angehört.

Beweis: Sei $F_{0}$ irgend eine Abbildung der $S^{k}$ in das Polyeder $P$, die der gegebenen Klasse angehört, und sei $f_{0}$ die Abbildung, die $F_{0}$ auf $E$ indu- 
ziert. Wir konstruieren eine stetige Abbildungsschar $f_{t}(0 \leqq t \leqq 1)$, die $f_{0}$ mit $f_{1}$ verbindet. (Eine derartige Schar läßt sich finden, da $P$ zusammenhängend ist.) Die Schar $f_{t}$ läßt sich zu einer Abbildungsschar $F_{t}$ der $S^{k}$ in das Polyeder $P$ erweitern $\left.{ }^{14}\right) ; F_{1}$ ist unsere gesuchte Abbildung.

Identifiziert man in diesem Hilfssatz $S^{k}$ mit unserem Zylinder $Z$ über einer Zelle $E^{n-m}$ (Nr. 3) und $P$ mit der Mannigfaltigkeit $V_{n, m}^{*}$, so ergibt sich leicht:

Satz 13. Ein auf der Zelle $E^{n-m}$ gegebenes stetiges $m$-Feld kann immer durch ein zweites Feld auf dieser Zelle zu einem Feldpaar mit starren Randwerten (Nr. 3) und vorgegebener Charakteristik ergänzt werden.

\section{§ 4. Vektorfelder in Mannigfaltigkeiten}

\section{1. $m$-Felder, Parallelisierbarkeit}

Wir gehen nun zum Studium von $m$-Feldern in einer geschlossenen $n$-dimensionalen und differenzierbaren Mannigfaltigkeit $M^{n}$ über. Dabei müssen wir vorläufig die Fallunterscheidung von Satz 9 durchführen:

Fall 1. $n-m$ ist gerade oder $m=1$. Dann sei $M^{n}$ orientierbar.

Fall 2. $n-m$ ist ungerade und $m \neq 1$. Dann kann $M^{n}$ auch nicht orientierbar sein.

$M^{n}$ nennen wir differenzierbar, wenn die folgende Bedingung erfüllt ist: $M^{n}$ ist mit einem ein für alle Mal fest gewählten System von Umgebungen, die wir im folgenden Elemente nennen, bedeckt. Jedes Element ist zum euklidischen Raum $R^{n}$ homöomorph und mit einem cartesischen Koordinatensystem ausgerüstet. Die durch das Übereinandergreifen von zwei Koordinatensystemen induzierte Koordinatentransformation soll stetig differenzierbar sein und eine nirgends verschwindende und im Fall 1 sogar positive Funktionaldeterminante besitzen.

Unter diesen Voraussetzungen kann man in der $M^{n}$ Vektoren definieren und auf sie die Begriffsbildungen und Sätze von $\S 3$ anwenden; man hat nur den euklidischen Raum sinngemäß durch ein Element in $M^{n}$ zu ersetzen.

Ist in jedem Punkte von $M^{n}$ ein $m$-System angebracht, so sprechen wir von einem $m-F$ eld in $M^{n}$; dieses Feld heißt stetig, falls es in jedem Element stetig ist. Gibt es in $M^{n}$ stetige $\mu$-Felder aber keine stetigen

14) AH: Kap. XIII, § 1, Hilfssatz Ia. 
$(\mu+1)$-Felder, so nennen wir $\mu$ den Grad der Parallelisierbarkeit von $M^{n}$; eine Mannigfaltigkeit mit $\mu=n$ wird als parallelisierbare Mannigfaltigkeit bezeichnet. Die Gründe für diese Bezeichnung sind leicht ersichtlich : Ist $\mu=n$, so gibt es in $M^{n}$ ein stetiges Basisfeld ( $\$ 3$, Nr. 2); legen wir einen beliebigen Vektor von $M^{n}$ mit dem Angriffspunkt $p$ durch seine Komponenten bezüglich der in $p$ vorliegenden Basis fest, so heißen eben zwei Vektoren parallel, wenn sie positiv-proportionale Komponenten besitzen. Damit ist in $M^{n}$ ein stetiger Fernparallelismus konstruiert, aus dem zum Beispiel folgt, daß die Mannigfaltigkeit der gerichteten Linienelemente der $M^{n}$ zum topologischen Produkt der $M^{n}$ mit einer $(n-1)$-dimensionalen Sphäre homöomorph ist. Beispiele parallelisierbarer Mannigfaltigkeiten sind leicht anzugeben : Das Produkt aus zwei parallelisierbaren Mannigfaltigkeiten ist wieder parallelisierbar, also liefert der $n$-dimensionale Torus (Produkt aus $n$ Kreislinien) ein Beispiel für eine parallelisierbare $M^{n}$. Wir bemerken noch, daß man in einer parallelisierbaren $M^{n}$ genau wie im euklidischen Raum Charakteristiken durch Parallelverschiebung aller beteiligten Vektoren an einen festen Punkt der $M^{n}$ berechnen kann ( $\$ 3$, Nr. 2).

Das zentrale Problem dieser Arbeit, zu dessen Lösung im folgenden einige Schritte getan werden, ist die Bestimmung des Grades $\mu$ einer vorgelegten Mannigfaltigkeit. Wir sind berechtigt, dieses Problem ein topologisches zu nennen, denn zwei Mannigfaltigkeiten, die sich vermöge einer ein-eindeutigen und in beiden Richtungen stetig differenzierbaren Abbildung entsprechen, haben offenbar denselben Grad.

\section{Gerüste und Gerüstpaare}

Den folgenden Betrachtungen sei eine feste Zellenzerlegung der $M^{n}$ zugrunde gelegt; eine $r$-dimensionale orientierte Zelle bezeichnen wir mit $x^{r}$, die zu $x^{r}$ duale Zelle der dualen Zellteilung $\left.{ }^{15}\right)$ mit $\xi^{n-r}$. Die Zellenzerlegung sei so fein, daß der Stern von $x^{r}$ (das ist die Gesamtheit aller Zellen, die mit $x^{r}$ Punkte gemeinsam haben) ganz in einem Element der $M^{n}$ liegt. Im Falle 1 (Nr. 1) wollen wir ferner die duale Zelle $\xi^{n-r} \mathrm{zu} x^{r}$ so orientieren, wie das in orientierbaren Mannigfaltigkeiten üblich ist.15); im Fall 2 werden Orientierungen überhaupt keine Rolle spielen.

Ein Gerüst ist nun ein auf allen Zellen eines Teilkomplexes $K$ der dualen Zellteilung definiertes und daselbst stetiges $m$-Feld. Ist $K$ homogen $\varrho$-dimensional ${ }^{16}$ ), so sprechen wir auch kurz von einem $\varrho$-dimen-

\footnotetext{
15) vgl. Seifert-Threlfall: Lehrbuch der Topologie (B. G. Teubner, 1934), ferner AH: Kap. XI, § $1 . \S 68$.

$\left.{ }^{16}\right)$ vgl. AH: Kap. IV, § 1, Nr. 2.
} 
sionalen Gerüst. In dem für uns wichtigsten Fall ist $K$ der Komplex aller $\varrho$-dimensionalen Zellen der dualen Zellteilung; ein zu diesem Komplex gehöriges Gerüst heißt ein in der ganzen Mannigfaltigkeit $M^{n}$ definiertes $\varrho$-dimensionales Gerüst. Im folgenden wird immer vorausgesetzt, daß die Zellen von $K$ höchstens $(n-m)$-dimensional sind.

Dann gilt:

Satz 14. Jedes Gerüst in $M^{n}$ kann zu einem in der ganzen Mannigfaltigkeit $M^{n}$ definierten $(n-m)$-dimensionalen Gerüst ergänzt werden. $(0<m<n)$.

Beweis: $\xi^{e}$ seien die Zellen von $K$ und $\bar{\xi}^{e}$ die Zellen der dualen Zellenzerlegung, die nicht zu $K$ gehören. Nun bringe man in jeder Ecke $\bar{\xi}^{0}$ ein beliebiges $m$-System an. Damit ist auf dem. Rand jeder Zelle $\bar{\xi}^{1}$ ein $m$-Feld gegeben, das nach Satz 11 stetig ins Innere fortgesetzt werden kann. Das nun auf dem Rand jeder Zelle $\bar{\xi}^{2}$ gegebene $m$-Feld kann wieder (falls $m<n-1$ ist) ins Innere der Zelle fortgesetzt werden. (Satz 11.) Durch Weiterführen der Konstruktion beweist man den Satz. Aus ihm folgt:

Korollar: Es gibt in der ganzen $M^{n}$ definierte $(n-m)$-dimensionale Gerüste.

Derartige Gerüste werden wir immer mit \& bezeichnen.

Unter $\dot{\mathfrak{S}}$ verstehen wir das Gerüst, das $\mathfrak{G}$ auf dem Komplex der $(n-m-1)$-dimensionalen Zellen der dualen Zellenzerlegung liefert, während ein beliebiges in der ganzen $M^{n}$ definiertes $(n-m-1)$ dimensionales Gerüst mit $\mathfrak{g}$ bezeichnet wird.

Zwei Gerüste $\mathfrak{G}_{0}$ und $\mathfrak{G}_{1}$ bilden ein Gerüstpaar, wenn eine stetige Schar $\mathfrak{g}_{t}(0 \leqq t \leqq 1)$ von Gerüsten $\mathfrak{g}$ mit $\mathfrak{g}_{0}=\dot{\mathfrak{G}}_{0}$ und $\mathfrak{g}_{1}=\dot{\mathfrak{G}}_{1}$ gegeben ist. $\mathfrak{G S}_{0}$ und $\mathfrak{G}_{1}$ sind also auf dem Komplex $\operatorname{der}(n-m-1)$-dimensionalen Zellen von $M^{n}$ durch eine stetige Schar miteinander verbunden.

Satz 15. Zwei beliebige Gerüste $\mathfrak{G}_{0}$ und $\mathfrak{S}_{1}$ können immer zu einem Gerüstpaar vereinigt werden.

Der Beweis erfolgt analog wie bei Satz 14. Man verbinde zunächst die beiden durch $\mathfrak{G}_{0}$ und $\mathfrak{S}_{1}$ in einer Ecke $\xi^{0}$ der dualen Zellteilung gegebenen $m$-Systeme durch eine stetige Schar von $m$-Systemen und setze dann diese Verbindung nach Satz 12 in die höherdimensionalen Zellen fort. 


\section{Vorbemerkungen über Charaktere in $\Lambda^{n-r}$.}

Wir wählen zunächst einen Koeffizientenring $J$, der uns zur Aufstellung algebraischer Komplexe in $M^{n}$ dienen wird ${ }^{17}$ ), und zwar sei $J$ im Fall 1 (Nr. 1) der Ring der ganzen Zahlen und im Fall 2 der Restllassenring $(\bmod 2)$. Algebraische Teilkomplexe der $x$-Zellenzerlegung bezeichnen wir mit $C$ und algebraische Teilkomplexe der $\xi$-Zellenzerlegung mit $\Gamma$. Alle $(n-r)$-dimensionalen Komplexe $\Gamma^{n-r}$ bilden eine Gruppe $\Lambda^{n-r}$, die als Untergruppen die Gruppe $Z^{n-r}$ der $(n-r)$-dimensionalen Zyklen und die Gruppe $H^{n-r}$ der $(n-r)$-dimensionalen Ränder enthält. Die Differenzgruppe $Z^{n-r}-H^{n-r}$ ist bekanntlich die $(n-r)$ dimensionale Betti'sche Gruppe $B^{n-r}$ von $M^{n}$.

Ein Charakter $\chi$ in $\Lambda^{n-r}$ ist eine homomorphe Abbildung von $\Lambda^{n-r}$ in den Koeffizientenring $J$. Sind also $\Gamma_{1}$ und $\Gamma_{2}$ Komplexe aus $\Lambda^{n-r}$ und $\alpha$ ein Element von $J$, so gilt:
a) $\chi\left(\Gamma_{1}+\Gamma_{2}\right)=\chi\left(\Gamma_{1}\right)+\chi\left(\Gamma_{2}\right)$;
b) $\chi\left(a \Gamma_{1}\right)=a \chi\left(\Gamma_{1}\right)$.

Aus diesen beiden Tatsachen folgt:

c) Ein Charakter $\chi$ ist bekannt, wenn seine Werte für die Komplexe einer Basis von $A^{n-r}$ gegeben sind. (Alle Zellen $\xi^{n-r}$ bilden z. B. eine derartige Basis.)

d) Ist $C$ ein festgewählter $r$-dimensionaler Teilkomplex der $x$-Zellenzerlegung, so wird durch die Festsetzung

$$
\chi(\Gamma)=\varnothing(C, \Gamma)
$$

ein Charakter $\chi$ in $\Lambda^{n-r}$ erzeugt. (Dabei ist $\Gamma$ ein beliebiger Komplex von $A^{n-r}$ und $\varnothing$ bedeutet die Schnittzahl der in der Klammer stehenden Komplexe.)

e) Jeder Charakter in $A^{n-r}$ kann durch einen Komplex $C$ auf die bei d) angedeutete Weise erzeugt werden. $C$ ist eindeutig bestimmt, und heißt der zu $\chi$ gehörige Komplex.

Der Beweis erfolgt am besten durch explizite Angabe von $C$. Es ist:

$$
C=\sum_{(j)} \chi\left(\xi_{j}^{n-r}\right) x_{j}^{r}
$$

Dabei ist die Summation über alle $r$-dimensionalen Zellen $x_{j}^{r}$ zu erstrecken.

17) AH: Kap. IV. 
Unser nächstes Ziel ist, Eigenschaften des erzeugenden Komplexes $C$ aus Eigenschaften des Charakters $\chi$ zu bestimmen:

f) $C$ ist dann und nur dann Zyklus, wenn $\chi$ in der Gruppe $H^{n-r}$ verschwindet, d. h. wenn für jeden $(n-r+1)$-dimensionalen Komplex $\Delta$ der $\xi$-Zellenzerlegung gilt:

$$
\chi(\dot{\Delta})=0 .
$$

Der Beweis folgt aus der Tatsache, daß für irgend einen Charakter $\chi$ und für beliebiges $\Delta$ die Beziehung

$$
\chi(\dot{\Delta})=\varnothing(C, \dot{\Delta})= \pm \varnothing(\dot{C}, \Delta)
$$

gilt.

g) Besteht zwischen zwei Charakteren $\chi_{0}$ und $\chi_{1}$ in $\Lambda^{n-r}$ und einem Charakter $\dot{\chi}$ in $\Lambda^{n-r-1}$ die Beziehung

$$
\chi_{1}(\Gamma)-\chi_{0}(\Gamma)=\dot{\chi}(\dot{\Gamma})
$$

so besteht zwischen den zugehörigen Komplexen $C_{0}, C_{1}$ und $D$ die Relation:

$$
C_{1}-C_{0}= \pm \dot{D}
$$

Beweis: Man hat für einen beliebigen $(n-r)$-dimensionalen Komplex $\Gamma$ :

$$
\begin{gathered}
\varnothing\left(C_{1}-C_{0}, \Gamma\right)=\varnothing\left(C_{1}, \Gamma\right)-\varnothing\left(C_{0}, \Gamma\right)=\chi_{1}(\Gamma)-\chi_{0}(\Gamma) \\
=\dot{\chi}(\dot{\Gamma})=\varnothing(D, \dot{\Gamma})= \pm \varnothing(\dot{D}, \Gamma) .
\end{gathered}
$$

Da $\Gamma$ beliebig war, folgt daraus die Behauptung:

h) Sei eine Menge von Charakteren $\chi_{i}$ in der Gruppe $\Lambda^{n-r}$ gegeben, von denen jeder die Bedingung (I) erfüllt, und von denen je zwei eine Beziehung von der Form (II) erfüllen. Dann gilt:

a) Die gegebene Menge bestimmt einen Charakter $\chi^{*}$ in der Betti'schen Gruppe $B^{n-r}$, deren Elemente (es sind dies Homologieklassen) wir mit $\Xi$ bezeichnen.

$\beta$ ) Die zu den $\chi_{i}$ gehörigen Komplexe sind nach f) Zyklen und liegen in einer einzigen $r$-dimensionalen Homologieklasse $A$.

r) Es gilt: $\chi^{*}(\Xi)=\varnothing(A, \Xi)$. 
Beweis:

$\mathrm{Zu} \alpha$ ): Aus dem Bestehen von (II) folgt zunächst, daß alle Charaktere $\chi_{i}$ in der Zyklengruppe $Z^{n-r}$ übereinstimmen und somit einen einzigen Charakter in dieser Gruppe induzieren. Wegen (I) hat überdies dieser Charakter für homologe Zyklen denselben Wert, bestimmt also tatsächlich einen Charakter $\chi^{*}$ in der Betti'schen Gruppe $B^{n-r}$.

$\mathrm{Zu} \beta$ ): Wegen (II) gilt die Behauptung von g), aus der folgt, daß die zu zwei Charakteren der gegebenen Menge gehörigen Zyklen homolog sind.

$\gamma$ ) folgt direkt aus den Definitionen von $\chi^{*}$ und $A$.

\section{Die dureh Gerüste bestimmten Charaktere; Hauptsätze}

Sei nun ein in der ganzen Mannigfaltigkeit $M^{n}$ definiertes $(n-m)$ dimensionales Gerüst $\mathfrak{S}$ gegeben. (Korollar zu Satz 14.) Wir definieren nun in $A^{n-m+1}$ einen Charakter $\chi$, indem wir nach Nr. 3, c) die Werte von $\chi$ für die Zellen $\xi^{n-m+1}$ geben: Es sei $\chi\left(\xi^{n-m+1}\right)$ die Charakteristik des durch $\mathfrak{G}$ auf der Randsphäre $\xi^{\dot{n}-m+1}$ von $\xi^{n-m+1}$ gegebenen stetigen $m$-Feldes. (Zur Berechnung dieser Charakteristik wird natürlich die Orientierung $\dot{\xi}^{n-m+1}$ verwendet. Man beachte ferner, daß die Charakteristik ein Element von $J$ ist.) Der so definierte Charakter heißt der $z u$ (S) gehörige Charakter $\chi$.

Wir betrachten weiterhin alle $(n-m)$-dimensionalen und in der ganzen $M^{n}$ definierten Gerüste $\mathfrak{G}_{i}$. Die zu ihnen gehörigen Charaktere $\chi_{i}$ bilden eine Menge, wie wir sie in Nr. $3 \mathrm{~h}$ ) betrachtet haben. Wir behaupten, daß diese Menge die Voraussetzungen von $\mathrm{Nr} .3 \mathrm{~h}$ ) erfüllt.

Beweis: Seien etwa $\mathfrak{G}_{0}$ und $\mathfrak{G}_{1}$ zwei Gerüste und $\chi_{0}$ und $\chi_{1}$ die zugehörigen Charaktere. Wir zeigen zunächst, daß ein Charakter $\dot{\chi}$ in $A^{n-m}$ existiert, so daß $\chi_{0}, \chi_{1}$ und $\dot{\chi}$ die Beziehung (II) von Nr. 3 erfüllen. Wegen Nr. 3 c) genügt es, $\dot{\chi}$ für die Zellen $\xi^{n-m}$ zu definieren. Zu diesem Zweck verbinden wir nach Satz 15 die Gerüste $\mathfrak{G}_{0}$ und $\mathfrak{G}_{1}$ zu einem Gerüstpaar; $\dot{\chi}\left(\xi^{n-m}\right)$ sei die Charakteristik des durch dieses Gerüstpaar auf $\xi^{n-m}$ gelieferten Feldpaares. Dann ist wegen der Formel $(C)$ von $\S 3$, Nr. 3:

$$
\chi_{1}\left(\xi^{n-m+1}\right)-\chi_{0}\left(\xi^{n-m+1}\right)=\dot{\chi}\left(\dot{\xi}^{n-m+1}\right) .
$$

Aus Nr.3a) und b) folgt nun in der Tat die Relation (II). Ferner haben wir zu zeigen, daß jeder unserer Charaktere der Bedingung (I) von Nr. 3 
genügt. Wenden wir die eben bewiesene Beziehung (II) auf den Komplex $\dot{\Delta}$ an, so ergibt sich:

$$
\chi_{1}(\dot{\Delta})=\chi_{0}(\dot{\Delta})
$$

es genügt also, (I) für einen einzigen durch ein spezielles Gerüst $\mathfrak{S}_{0}$ gelieferten Charakter zu beweisen. Weiterhin genügt es wegen a) und b), $\mathrm{daß} \Delta$ eine Zelle $\xi^{n-m+2}$ ist. Wir konstruieren nun $\mathfrak{G}_{0}$ folgendermaßen: Auf dem Rand $\dot{\xi}^{n-m+2}$ seien die $m$-Systeme von $\mathfrak{G}_{0}$ zu einander parallel. (Diese Definition hat einen Sinn, da $\xi^{n-m+2}$ in einem Element (Nr. 1) liegt.) Ein derartiges Gerüst kann nach Satz 14 immer gefunden werden. Für den zugehörigen Charakter $\chi_{0}$ gilt nun trivialerweise $: \chi_{0}\left(\dot{\xi}^{n-m+2}\right)=0$, womit $(\mathrm{I})$ in allen Teilen bewiesen ist.

Aus den Behauptungen von Nr. 3, h) folgt nun:

Der zu einem Gerüst $\mathfrak{S}$ gehörige Charakter $\chi$ besitzt als zugehörigen Komplex einen Zyklus, der singulärer Zyklus von $\mathfrak{G}$ genannt wird und nach Nr. 3, e) durch

$$
z=\underset{(j)}{\sum_{\chi}} \chi\left(\xi_{j}^{n-m+1}\right) x_{j}^{m-1}
$$

gegeben ist. Sämtliche Charaktere $\chi_{i}$ bestimmen einen Charakter $\chi^{*}$ in der $(n-m+1)$-dimensionalen Betti'schen Gruppe $B^{n-m+1}$, den wir im folgenden $\chi^{n-m+1}$ nennen ${ }^{18}$ ). Ferner gilt:

Satz 16. (Erster Hauptsatz.) Die singulären Zyklen aller $(n-m)$ dimensionalen und aus m-Systemen bestehenden Gerüste $\mathfrak{G}$, die in der ganzen Mannigfaltigkeit $M^{n}$ definiert werden können, liegen in einer einzigen $(m-1)$-dimensionalen Homologieklasse; sie heißt die charakteristische Homologieklasse $F^{m-1}$. Ist $\Xi$ eine beliebige $(n-m+1)$-dimensionale Homologieklasse, so gilt: $\chi^{n-m+1}(\Xi)=\varnothing\left(F^{m-1}, \Xi\right)$.

Wir werden im nächsten Paragraphen sehen, daß der Charakter $\chi^{n-m+1}$ eine Verallgemeinerung der Euler'schen Charakteristik darstellt.

Diesen unmittelbaren Folgerungen aus den Betrachtungen der Nr. 3 müssen wir noch den folgenden, etwas tiefer liegenden Satz hinzufügen:

Satz 1\%. (Zweiter Hauptsatz.) Jeder in der charakteristischen Klasse $F^{m-1}$ enthaltene $Z y k l u s$ ist singulärer Zyklus eines Gerüstes.

18) Der Charakter $\%^{\mu-i n+1}$ ist ex definitione unabhängig von der Wahl eines Gerüstes; er ist durch die geometrischen Eigenschaften von $M^{n}$ gegeben. 
Beweis: Sei etwa $z$ der gegebene Zyklus in der Klasse $F^{m-1}$. Wir wählen ein beliebiges aber festes Ausgangsgerüst $\mathfrak{G}_{0}$ mit dem singulären Zyklus $z_{0}$. Nach Satz 16 liegt $z_{0}$ auch in $F^{m-1}$, es ist also $z \sim z_{0}$, und somit $z-z_{0}=\dot{D}$. Unser Gerüst $\mathfrak{G}_{0}$ liefert auf der Zelle $\xi^{n-m}$ ein $m$-Feld $\mathscr{f}_{0}$, das wir durch ein weiteres Feld $\mathfrak{F}_{1}$ zu einem Feldpaar mit starren Randwerten ergänzen ( $(3, \mathrm{Nr} .3)$, dessen Charakteristik auf $\xi^{n-m}$ den Wert $\varnothing\left(D, \xi^{n-m}\right)$ besitzt (Satz 13). Die so auf allen Zellen $\xi^{n-m}$ konstruierten $m$-Felder $\mathscr{F}_{1}$ schließen sich zu einem Gerüst $\mathfrak{G}_{1}$ zusammen. $\mathfrak{S}_{0}$ und $\mathfrak{G}_{1}$ bilden zusammen ein Gerüstpaar, das wie am Anfang dieser Nummer Anlaß zu einem Charakter $\dot{\chi}$ gibt. Es ist nach Konstruktion $\dot{\chi}\left(\xi^{n-m}\right)=\varnothing\left(D, \xi^{n-m}\right)$, d. h. der zum Charakter $\dot{\chi}$ gehörige Komplex ist der Komplex $D$.

Wir haben gesehen, daß zwischen den Charakteren $\chi_{0}$ von $\mathfrak{S}_{0}$ und $\chi_{1}$ von $\mathfrak{G}_{1}$ und dem Charakter $\dot{\chi}$ die Relation (II) von Nr. 3 besteht, es gilt also die Behauptung von Nr. 3,g), d. h. $z_{1}-z_{0}= \pm \dot{D}$, falls wir den singulären Zyklus von $\mathbb{S}_{1}$ mit $z_{1}$ bezeichnen. Aus dieser Beziehung (in der etwa das Pluszeichen gelten soll) und aus $z-z_{0}=\dot{D}$ folgt $z=z_{1}$. Der gegebene Zyklus $z$ ist also singulärer Zyklus von $\mathfrak{G}_{1}$, womit Satz 17 bewiesen ist.

Die Bedeutung der charakteristischen Klasse $F^{m-1}$ für das Problem dieser Arbeit beruht auf dem folgenden Satz:

Satz 18. (Existenzsatz.) Es gibt dann und nur dann in der ganzen Mannigfaltigkeit $M^{n}$ definierte $(n-m+1)$-dimensionale Gerüste, wenn die charakteristische Klasse $F^{m-1}$ die 0-Klasse ist.

Beweis: a) Sei ein in der ganzen $M^{n}$ definiertes $(n-m+1)$-dimensionales Gerüst gegeben. Es liefert auf dem Komplex der Zellen $\xi^{n-m}$ ein Gerüst $\mathfrak{G}$, also auf jedem Zellenrand $\dot{\xi}^{n-m+1}$ ein $m$-Feld $\mathfrak{F}$. Da $\mathfrak{F}$ ins Innere der Zelle $\xi^{n-m+1}$ fortgesetzt ist, verschwindet seine Charakteristik auf $\dot{\xi}^{n-m+1}$, also verschwindet auch der zu $\mathfrak{S}$ gehörige Charakter $\chi$, und für den singulären Zyklus $z$ von $\mathfrak{G}$ hat $\operatorname{man} z=0$, also erst recht $z \sim 0$.

b) Sei die charakteristische Klasse $F^{m-1}$ die 0-Klasse. Nach Satz 17 gibt es ein Gerüst $\mathfrak{G}$, dessen singulärer Zyklus der 0-Zyklus ist. Also verschwindet der zu $\&$ gehörige Charakter $\chi$; dann kann aber nach Satz 11 das durch \& auf $\dot{\xi}^{n-m+1}$ gelieferte Feld ins Innere fortgesetzt werden. 


\section{Felder mit Singularitäten}

Unsere Bemühungen, in der Mannigfaltigkeit $M^{n}$ schrittweise durch Gerüste ein stetiges $m$-Feld zu konstruieren, sind an der Existenz der Klasse $F^{m-1}$ gescheitert; wir können aber immer $m$-Felder finden, bei denen die Stetigkeit in gewissen ,,singulären“ Punkten durchbrochen ist. Um nicht in dimensionstheoretische Schwierigkeiten zu geraten, wollen wir nur $m$-Felder betrachten, die der folgenden Voraussetzung genügen: Enthält eine Zelle $x^{r-1}$ unserer $x$-Zellenzerlegung einen singulären Punkt im Innern, so besteht sie aus lauter singulären Punkten. Alle diese Zellen bilden einen absoluten Komplex $K^{r-1}$, den Singularitätenkomplex des betreffenden Feldes. [Die Zahl $(r-1)$ bedeutet die Dimension der höchstdimensionalen Zelle in diesem Komplex.] Ein Feld mit dem Singularitätenkomplex $K^{r-1}$ liefert nun offenbar ein $(n-r)$-dimensionales und in der ganzen $M^{n}$ definiertes Gerüst. Es gilt aber auch die Umkehrung: $\mathrm{Zu}$ jedem $(n-r)$-dimensionalen und in der ganzen $\boldsymbol{M}^{n}$ definierten Gerüst gehört ein $m$-Feld in der $M^{n}$ mit einem Singularitätenkomplex $K^{r-1}$. Um dies einzusehen, setze man das durch das Gerüst auf den Zellen $\xi^{n-r}$ gegebene $m$-Feld durch Zentralprojektion ( $(3, \mathrm{Nr} .1)$ in die höherdimensionalen Zellen $\xi^{n-r+k}$ fort. Wählt man dabei als Projektionszentrum den Schnittpunkt von $\xi^{n-r+k}$ mit der dualen Zelle $x^{r-k}$, so lassen sich die nötigen Kegelkonstruktionen in einer gemeinsamen Unterteilung $U$ der $x$ - und $\xi$-Zellenzerlegung simplizial durchführen. Man erhält so ein Feld, dessen Singularitätenkomplex ein simplizialer Teilkomplex von $U$ ist. Durch diese Beziehung zwischen Gerüsten und singulären Feldern folgt nun aus dem Korollar zu Satz 14 und aus Satz 18:

Satz 19. In einer Mannigfaltigkeit $M^{n}$ gibt es immer ein $m-F$ eld mit einem $(m-1)$-dimensionalen Singularitätenkomplex; die notwendige und hinreichende Bedingung für die Existenz eines m-Feldes mit einem höchstens $(m-2)$-dimensionalen Singularitätenkomplex ist das Verschwinden der charakteristischen Klasse $F^{m-1}$.

$\mathrm{Da}$ jedes singuläre $m$-Feld in $M^{n}$ mit einem $(m-1)$-dimensionalen Singularitätenkomplex ein $(n-m)$-dimensionales und in der ganzen $M^{n}$ definiertes Gerüst $\mathfrak{S}$ eindeutig bestimmt, können wir den zu $\mathfrak{S}$ gehörigen singulären Zyklus (Nr. 4) kurz den singulären Zyklus des gegebenen Feldes nennen. Es gilt dann:

Satz 19 a. Der singuläre Zyklus eines $m$-Feldes mit dem $(m-1)$ dimensionalen Singularitätenkomplex $K^{m-1}$ ist in seiner durch $U$ gelieferten Unterteilung algebraischer Teilkomplex von $K^{m-1}$; er mißt die Vielfachheit 
der $(m-1)$-dimensionalen Singularitäten und repräsentiert die charakteristische Klasse $F^{m-1}$.

Zum Beweise verwende man die explizite Darstellung des singulären Zyklus $z=\underset{(j)}{ } \chi\left(\xi_{j}^{n-m+1}\right) x_{j}^{m-1}$ und Satz 11 .

\section{$\S 5$. Bestimmung der charakteristischen Klasse in speziellen Fällen}

\section{Differenzierbare Simplizialzerlegungen}

Eine Simplizialzerlegung $K$ der vorgelegten Mannigfaltigkeit heißt differenzierbar, wenn jedes Simplex von $K$ samt seinem Stern in einem Element der $M^{n}$ (§ 4, Nr. 1) liegt und in diesem Element ein euklidisches Simplex ${ }^{19}$ ) oder das Bild von einem euklidischen Simplex vermöge einer topologischen und in beiden Richtungen stetig differenzierbaren Abbildung ist.

Für das Folgende brauchen wir die baryzentrische Unterteilung ${ }^{20}$ ) $\breve{K}$ einer derartigen Simplizialzerlegung $K$. Bezeichnen wir den Schwerpunkt eines $r$-dimensionalen Simplexes von $K$ mit $a_{r}$, so sind die Simplexe $x^{s}=\left(a_{r_{0}}, a_{r_{1}}, \ldots, a_{r_{g}}\right)$ die Simplexe von $\bar{K} .\left(r_{0}<r_{1}<\ldots r_{s}\right)$ und $(s=0,1, \ldots, n) . \bar{K}$ sei nun unsere $x$-Zellenzerlegung von $\S 4$; die duale Zelle $\xi^{n-s}$ von $x^{s}$ bezeichnen wir mit $\xi^{n-s}=\xi_{\left(r_{0} r_{1} \ldots r_{8}\right)}$.

\section{Einzelne Vektorfelder}

Wir beschäftigen uns in dieser Nummer mit der Theorie der 1-Felder (im folgenden kurz als Vektorfelder bezeichnet) in einer Mannigfaltigkeit $M^{n}$. Diese Theorie ist schon vor längerer Zeit entwickelt worden ${ }^{21}$ ), die abschließenden Resultate (Sätze 20 und 20a) stammen von $H$. Hopf.

Satz 19 zeigt zunächst, daß es in $M^{n}$ immer ein Vektorfeld $\mathscr{F}$ mit 0-dimensionalem Singularitätenkomplex gibt; $\mathfrak{F}$ ist also nur in endlich vielen Ecken $x_{i}^{0}$ der $x$-Zellenzerlegung singulär. Unter dem Index $j_{i}$ der Singularität $x_{i}^{0}$ versteht man die Charakteristik des durch $\mathscr{f}$ auf dem Rand $\dot{\xi}_{i}^{n}$ der zu $x_{i}^{0}$ dualen Zelle $\xi_{i}^{n}$ gegebenen 1-Feldes. (Wir befinden uns im Fall 1 von $\S 4$, Nr. $1 ; M^{n}$ ist daher orientierbar, und die Zellen $\xi_{i}^{n}$ seien kohärent orientiert). Eine leichte Überlegung liefert für den singulären Zyklus von $\mathfrak{F}(\S 4, \mathrm{Nr} .5)$ :

$$
z=\sum_{(i)} j_{i} x_{i}^{\mathbf{0}}
$$

19) AH: Kap. III, \& 1, Nr. 1.

20) AH: Kap. III, $\S 2$, Nr. 3.

21) AH: Kap. XIV, § 4. 
Die charakteristische Klasse $F^{0}$ wird also durch den Zyklus $x^{0} \Sigma j_{i}$ repräsentiert ( $x^{0}$ ist eine beliebige, aber fest gewählte Ecke der $x$-Zellenzerlegung). Die Indexsumme $\Sigma j_{i}$ heißt algebraische Anzahl der Singularitäten. Bezeichnet man die durch die Summe sämtlicher Zellen $\xi^{n}$ repräsentierte $n$-dimensionale Homologieklasse mit $\Xi^{n}$, so ergibt sich für den Charakter $\chi^{n}$ in der Betti'schen Gruppe $B^{n}(\S 4, \mathrm{Nr} .4)$ nach Satz 16:

$$
\chi^{n}\left(\Xi^{n}\right)=\varnothing\left(F^{0}, \Xi^{n}\right)=\Sigma j_{i} .
$$

Da $\Xi^{n}$ einziges Basiselement von $B^{n}$ ist, bestimmt (2) den Charakter $\chi^{n}$ vollständig.

Aus Satz 16 und 18 folgt nun:

Satz 20. Die algebraische Anzahl der Singularitäten ist für alle Vektorfelder in $M^{n}$ dieselbe; es gibt dann und nur dann Vektorfelder, die in allen Punkten der $M^{n}$ stetig sind, wenn diese Anzahl verschwindet.

Ferner gilt:

Satz 20a. Bei geeigneter Orientierung von $M^{n}$ ist die algebraische Anzahl der Singularitäten jedes Vektorfeldes in $M^{n}$ gleich der Euler'schen Charakteristik $\chi\left(M^{n}\right)$ von $M^{n}$.

Mit diesem Satz ist die folgende Behauptung äquivalent:

Die charakteristische Klasse $F^{0}$ wird durch $x^{0} \cdot \chi\left(M^{n}\right)$ repräsentiert. Weiterhin besagt die Formel

$$
\chi^{n}\left(\Xi^{n}\right)=\chi\left(M^{n}\right)
$$

auch genau dasselbe. In dieser letzten Form werden wir den Satz für den einfachsten Fall $n=2$ beweisen. Wir führen den Beweis unter der Voraussetzung, da $B M^{2}$ eine differenzierbare Simplizialzerlegung besitzt. (Satz 20a ist auch ohne diese Voraussetzung richtig.) Dann konstruieren wir in der zur baryzentrischen Unterteilung dualen Zellenzerlegung ein spezielles eindimensionales und aus 1-Systemen bestehendes Gerüst $\mathfrak{S}$, dessen zugehörigen Charakter $\chi$ wir bestimmen. In Figur 3 ist der auf einem Simplex $\left(a_{0}, a_{1}, a_{2}\right)$ (Nr. 1) der baryzentrischen Unterteilung $\bar{K}$ liegende Teil von $\mathbb{S}$ gezeichnet. Aus dieser Figur ist ersichtlich, daß die Vektoren von $\mathfrak{G}$ auf dem Rand einer Zelle vom Typus $\xi_{(0)}$ (Nr. 1) ins Äußere von $\xi_{(0)}$ und auf dem Rand einer Zelle vom Typus $\xi_{(2)}$ ins Innere 
von $\xi_{(2)}$ zeigen. [In Fig. 3 sind die in $\left(a_{0}, a_{1}, a_{2}\right)$ liegenden Teile von drei Zellen $\xi_{(0)}, \xi_{(1)}, \xi_{(2)}$ angedeutet.] Bei geeigneter Orientierung findet man für die Charakteristik des durch $\mathfrak{G}$ auf dem Rand einer Zelle $\xi_{(r)}$ gelieferten Feldes den Wert $(-1)^{r}$, also hat man:

$$
\chi\left(\xi_{(r)}\right)=(-1)^{r} . \quad(r=0,1,2),
$$

und der singuläre Zyklus $z$ von $\mathfrak{S}$ wird:

$$
z=\Sigma(-1)^{r} a_{r},
$$

wobei die Summation über alle Ecken von $\bar{K}$ zu erstrecken ist. Bezeichnet man die Anzahl der Zellen vom Typus $\xi_{(r)}$ mit $\alpha_{r}$, so ergibt sich demnach für den Charakter $\chi^{n}=\chi^{2}$ :

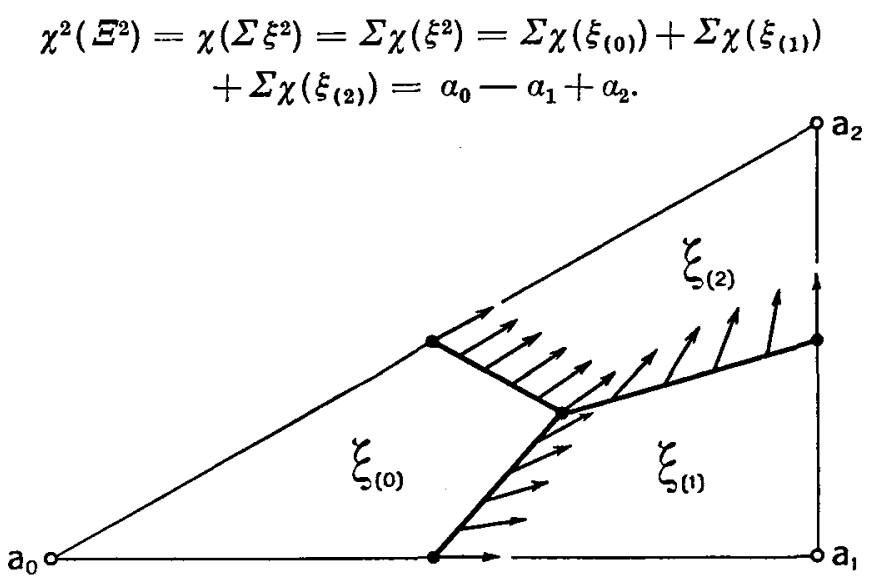

Fig. 3

$\alpha_{0}-a_{1}+\alpha_{2}$ ist aber definitionsgemäß die Euler'sche Charakteristik $\chi\left(M^{2}\right)$; damit ist (3) im Spezialfall $n=2$ bewiesen. In analoger Weise läßt sich Satz 20 a) für $n$-dimensionale Mannigfaltigkeiten beweisen.

Die Formel (3) belegt die in $\$ 4$ erwähnte Tatsache, daß der Charakter $\chi^{n-m+1}$ als Verallgemeinerung der Euler'schen Charakteristik aufgefaßt werden kann.

Aus den Sätzen 20 und 20 a) folgt:

Korollar. In der Mannigfaltigkeit $M^{n}$ gibt es dann und nur dann ein stetiges Vektorfeld, wenn die Euler'sche Charakteristik $\chi\left(M^{n}\right)$ verschwindet ${ }^{21}$ ).

21 a) vgl. AH: Kap. XIV, § 4, Satz 3. 
Dieser Satz gilt auch für nichtorientierbare Mannigfaltigkeiten, ist aber mit unseren. Methoden nicht direkt beweisbar. Unsere Überlegungen lassen sich jedoch auch für nichtorientierbare Mannigfaltigkeiten durchführen, falls wir an Stelle des Ringes der ganzen Zahlen den Restklassenring $(\bmod 2)$ als Koeffizientenbereich einführen $(\S 4, \mathrm{Nr} .3)$. Verstehen wir in diesem Fall unter $\Xi^{n}$ die durch die Summe der (nichtorientierten) Zellen $\xi^{n}$ repräsentierte $n$-dimensionale Homologieklasse $(\bmod 2)$, so gilt:

$$
\chi^{n}\left(\Xi^{n}\right) \equiv \chi\left(M^{n}\right) \quad(\bmod 2) .
$$

\section{Dreidimensionale Mannigfaltigkeiten}

Wir untersuchen nun die Parallelisierbarkeit (§ 4, Nr. 1) der dreidimensionalen Mannigfaltigkeiten. Es ergibt sich das wichtige Resultat:

Sat: 21. Jede orientierbare dreidimensionale geschlossene Mannigfaltigkeit, die eine differenzierbare Simplizialzerlegung erlaubt, ist parallelisierbar.

Bevor wir den Beweis dieses Satzes angeben, erwähnen wir das aus den Betrachtungen von $\S 4, \mathrm{Nr} .1$ folgende

Korollar. Erfullt eine dreidimensionale Mannigfaltigkeit $M^{3}$ die Voraussetzungen von Satz 21, so ist die Mannigfaltigkeit ihrer gerichteten Linienelemente zum topologischen Produkt von $M^{3}$ mit einer zweidimensionalen Sphäre homöomorph.

Der Beweis von Satz 21 erfolgt in vier Schritten:

I. Bestimmung der charakteristischen Klasse $F^{\mathbf{1}}$.

Wir können uns zur Lösung dieser Aufgabe mit den folgenden Andeutungen begnügen, da wir imAnhang $I$ die charakteristische Klasse $F^{1}$ in dreidimensionalen orientierbaren Mannigfaltigkeiten unter etwas anderen Voraussetzungen und mit anderen Methoden ausführlich bestimmen werden.

$F^{1}$ ist die charakteristische Klasse der 2-Felder, wir haben also $m=2$ und $n=3$ zu setzen. Es liegt der Fall 2 von $\S 4$, Nr. 1 vor; $J$ ist somit der Restklassenring $(\bmod 2)$. Um $F^{1} \mathrm{zu}$ bestimmen, kann man auf analoge Weise wie in Nr. 2 (Fig. 3) ein spezielles 1-dimensionales in der ganzen $M^{3}$ definiertes Gerüst $\mathfrak{S}$ konstruieren, das mit der baryzentrischen Unterteilung $\bar{K}$ verknüpft ist. Ich gehe hier nicht auf die etwas mühsame 
Konstruktion dieses aus 2-Systemen bestehenden Gerüstes ein; man findet für den zugehörigen Charakter $\chi$ :

$$
\chi\left(\xi_{\left(r_{0} r_{1}\right)}\right)=1 \text {, }
$$

so daß also der singuläre Zyklus $z$ von $\mathfrak{G}$ durch

$$
z=\Sigma\left(a_{r_{0}}, a_{r_{1}}\right)
$$

gegeben ist $\left.{ }^{22}\right)$. Dieser Zyklus ( $\left.\bmod 2\right)$ besteht also aus sämtlichen Kanten der baryzentrischen Unterteilung $\bar{K}$. Man kann nun zeigen, daß $z$ in einer orientierbaren Mannigfaltigkeit $M^{3}$ immer berandet, während dies in nichtorientierbaren Mannigfaltigkeiten nicht notwendigerweise der Fall zu sein braucht ${ }^{23}$ ). Es ergibt sich also, daß in einer orientierbaren $M^{3}$ die charakteristische Klasse $F^{1}$ immer die 0 -Klasse ist.

II. Es existiert ein in der ganzen $M^{3}$ definiertes 2-dimensionales und aus 2-Systemen bestehendes Gerüst $\mathfrak{F}$.

Da nach $I$ in unserer orientierbaren $M^{3}$ die Klasse $F^{1}$ verschwindet, ist diese Tatsache eine direkte Folge des Existenzsatzes 18.

III. Es gibt in der $M^{3}$ stetige 2-Felder.

Um dies zu beweisen, zeigen wir, daß das durch $\mathfrak{S}$ auf dem Rande $\dot{\xi}^{3}$ einer Zelle $\xi^{3}$ gegebene 2-Feld $\mathfrak{F}$ sich stetig ins Innere von $\xi^{3}$ fortsetzen läßt. Da $\xi^{3}$ in einem Element $(\S 4, \mathrm{Nr} .1)$ der $M^{3}$ liegt, müssen wir daher den folgenden Satz beweisen: Ein auf der Randsphäre $S^{2}$ der im euklidischen Raum $R^{3}$ liegenden 3-dimensionalen Zelle $E^{3}$ gegebenes stetiges 2-Feld $\mathfrak{F}$ läßt sich stetig ins Innere von $E^{3}$ fortsetzen.

$\mathrm{Zu}$ diesem Satz ist die folgende Behauptung äquivalent: Die zu $\mathfrak{F}$ gehörige Abbildung der $S^{2}$ in die Mannigfaltigkeit $V_{3,2}^{*}$ durch parallele 2-Systeme ( $\$ 3, \mathrm{Nr} .1$ ) ist 0-homotop. Unsere Behauptung III läßt sich daher in der folgenden Form aussprechen: Jede stetige Abbildung einer 2-dimensionalen Sphäre $S^{2}$ in die $V_{3,2}^{*}$ ist 0-homotop. Da nun nach $\S 2$ Nr. 2 die geschlossene Mannigfaltigkeit $V_{3,2}$ Deformationsretrakt von

22) Die Formeln (4a) und (5a) legen die Vermutung nahe, daß für beliebiges $n$ und $m$ die charakteristische Klasse $F^{m-1}$

$$
\begin{aligned}
& \text { im Fall I von } \S 4 \text {, Nr. } 1 \text { durch } \Sigma(-1)^{r_{0}+r_{1}+\cdots+r_{m-1}}\left(a_{r_{0}}, a_{r_{2}}, \cdots, a_{r_{m-1}}\right) \\
& \text { und im Fall II durch } \Sigma\left(a_{r_{0}}, a_{r_{1}}, \cdots, a_{r_{m-1}}\right)
\end{aligned}
$$

repräsentiert wird. Die Summation ist dabei über alle $(m-1)$-dimensionalen Zellen von $\bar{K}$ zu erstrecken; die obigen Komplexe sind tatsächlich Zyklen des Koeffizientenbereiches $J$.

$\left.{ }^{23}\right)$ vgl. die Aufgabe $187 \mathrm{im}$ Jahresbericht der deutschen Mathematikervereinigung Band 45, S. 22. 
$V_{3,2}^{*}$ ist, genügt es, diese Behauptung für Abbildungen der $S^{2}$ in die $V_{3,2}$ zu beweisen. Da aber $V_{3,2}$ zum projektiven Raum $P^{3}$ homöomorph ist $\left(\S 1, \mathrm{Nr} .3\right.$ ), und da jede Abbildung einer $S^{2}$ in den $P^{3}$ in der Tat 0-homotop ist, haben wir die Behauptung III bewiesen.

IV. Es gibt stetige 3-Felder in der $M^{3}$.

Daß aus der Existenz stetiger 2-Felder in einer orientierbaren $M^{3}$ die Existenz stetiger 3-Felder folgt, ist leicht beweisbar.

\section{§ 6. Sätze aber die charakteristische Homologieklasse. Anwendungen}

\section{Ordnung der charakteristischen Klasse}

Wir stellen uns in diesem Abschnitt die Aufgabe, die Ordnung einer nicht verschwindenden charakteristischen Klasse zu bestimmen. Diese Aufgabe hat nur im Fall 1 von $\S 4$, Nr. 1 einen Sinn, in dem der Koeffizientenbereich $J$ der Ring der ganzen Zahlen ist. Wir werden sie für gerades $(n-m)$ lösen.

Wir schicken den folgenden Überlegungen eine Hilfsbetrachtung voraus, die sich auf die Mannigfaltigkeiten $V_{n, m}^{*}(\S 2)$, für die $n-m$ gerade ist, bezieht. Es soll nämlich die topologische Abbildung $\varphi$ der $V_{n, m}^{*}$ auf sich untersucht werden, die entsteht, wenn man in jedem $m$-System der $V_{n, m}^{*}$ den $m$-ten Vektor $\mathfrak{v}_{m}$ durch seinen entgegengesetzten Vektor $-\mathfrak{v}_{m}$ ersetzt. In der $(n-m)$-dimensionalen Sphäre, die in einer festen Orientierung den Basiszyklus $z_{n, m}$ von $\S 1$, Nr. 4 liefert, ist $\varphi$ die Diametralabbildung; da diese Sphäre eine gerade Dimension besitzt, ergibt sich:

$$
\varphi\left(z_{n, m}\right)=-z_{n, m} \text {. }
$$

Nach dieser Vorbereitung werde unter Verwendung der Bezeichnungen und Voraussetzungen von $\S 4$ in der gegebenen Mannigfaltigkeit $M^{n}$ ein aus $m$-Systemen bestehendes Gerüst $\mathbb{S}$ konstruiert, und es sei $n-m$ gerade. Wir befinden uns daher im Fall 1 von $\S 4$, Nr. 1 und der Koeffizientenbereich $J$ ist der Ring der ganzen Zahlen. Das Gerüst $\mathfrak{S}$ liefert auf dem Rande jeder $(n-m+1)$-dimensionalen Zelle $\xi$ ein $m$-Feld dessen Charakteristik $\chi(\xi)$ vermittelst der Abbildung $f$ durch parallele $m$-Systeme $(\S 3, \mathrm{Nr} .1)$ von $\dot{\xi}$ in die $V_{n, m}^{*}$ ermittelt wird.

Ersetzt man nun in jedem $m$-System von $\mathbb{S}$ den $m$-ten Vektor durch seinen entgegengesetzten Velktor, so entsteht ein neues Gerüst $\mathbb{E}$, zu dem die Charakteristik $\bar{\chi}(\xi)$ und die Abbildung $\bar{f}$ gehöre. Offenbar entsteht $\bar{f}$ 
durch die Zusammensetzung von $f$ und $\varphi$; aus (1) folgt daher: $\bar{\chi}(\xi)=-\chi(\xi)$. Zwischen den zu $\&$ und $\mathbb{E}$ gehörenden Charakteren $\chi$ und $\bar{\chi}$ besteht somit die Beziehung:

$$
\bar{x}=-\chi .
$$

Sowohl $\chi$ als auch $\bar{\chi}$ liefert in der $(n-m+1)$-dimensionalen Betti'schen Gruppe den Charakter $\chi^{n-m+1}$; aus (2) folgt daher: $\chi^{n-m+1}=-\chi^{n-m+1}$, und endlich $\chi^{n-m+1}=0$.

Es wäre falsch, aus dem Verschwinden von $\chi^{n-m+1}$ auf das Verschwinden der charakteristischen Klasse $F^{m-1}$ zu schließen; dieser Schluß ist nur zulässig, wenn in $M^{n}$ keine $(m-1)$-dimensionale Torsion vorhanden ist.

Setzen wir etwa $m=1$, so finden wir $\chi^{n}=0$ in Mannigfaltigkeiten ungerader Dimension; daraus folgt aber nach $\S 5$, Formel (3), daß die Charakteristik einer orientierbaren Mannigfaltigkeit ungerader Dimension verschwindet $\left.{ }^{24}\right)$. Aus dem Korollar zu Satz 20 folgt dann weiterhin, daß jede orientierbare Mannigfaltigkeit ungerader Dimension ein stetiges Vektorfeld besitzt.

Satz 22. Ist $M^{n}$ orientierbar, $(n-m)$ gerade, und ist die Klasse $F^{m-1}$ von der 0-Klasse verschieden, so besitzt sie die Ordnung 2.

Beweis: Wir haben zu zeigen: Für gerades $(n-m)$ ist immer $2 \cdot F^{m-1}=0$. Nun besteht wegen (2) zwischen den singulären Zyklen $z$ und $\bar{z}$ der oben verwendeten Gerüste $\mathfrak{S}$ und $\overline{\mathfrak{S}}$ die Relation $z=-\bar{z}$. Da diese Zyklen beide in $F^{m-1}$ liegen, ist $F^{m-1}=-F^{m-1}$; dies war zu beweisen.

Korollar. Ist $(n-m)$ gerade, und ist in $M^{n}$ keine $(m-1)$-dimensionale Torsion vorhanden, so ist $F^{m-1}$ die 0-Klasse.

\section{Ein Schnittsatz}

Im folgenden werden die Fälle 1 und 2 von $\S 4$, Nr. 1 nicht mehr unterschieden; sämtlichen Betrachtungen wird der Restklassenring $(\bmod 2)$ als Koeffizientenbereich $J$ zugrunde gelegt, und $M^{n}$ kann eine orientierbare oder nichtorientierbare Mannigfaltigkeit sein.

Um unsere Theorie bis zu einem gewissen Abschluß zu bringen, müssen wir Mannigfaltigkeiten finden, in denen von 0 verschiedene charakte-

24) Meines Wissens hat $J$. Hadamard zum erstenmal das Verschwinden der Euler'schen Charakteristik in einer Mannigfaltigkeit ungerader Dimensionszahl aus der Theorie der Vektorfelder hergeleitet. Vgl. Tannery: Introductión à la théorie des fonctions (Paris, Hermann, 1910), t. II, note de M. Hadamard, no. 42, p. 475. 
ristische Klassen existieren; nur dann enthalten die Sätze von $\S 4$ nichttriviale Aussagen. Die Ausführungen dieses Abschnitts dienen zur Lösung dieser Aufgabe.

Wir nennen eine in die gegebene Mannigfaltigkeit $M^{n}$ eingebettete $v$-dimensionale Mannigfaltigkeit $M^{\nu}$ eine $H$ yperfläche, wenn die folgenden Bedingungen erfüllt sind:

a) $M^{v}$ sei das Bild einer differenzierbaren Parametermannigfaltigkeit vermöge einer topologischen und stetig differenzierbaren Abbildung dieser Parametermannigfaltigkeit in die $M^{n}$.

b) $M^{\nu}$ gestatte eine Zellenzerlegung, die ein Teilkomplex der $\xi$-Zellenzerlegung $(\$ 4, \mathrm{Nr} .2)$ der Mannigfaltigkeit $M^{n}$ ist.

Wegen a) sind Vektoren in $M^{v}$ auch Vektoren in $M^{n}$, und die Gesamtheit aller Vektoren von $M^{v}$, die in einem Punkt $p$ von $M^{v}$ angreifen, bildet ein $\nu$-dimensionales Vektorgebilde von $M^{n}$. Gehören die Vektoren eines in $p$ angreifenden $(n-v)$-Systems der $M^{n}$ diesem Gebilde nicht an, so nennen wir das System fremd zu $M^{\nu}$. Existiert auf $M^{v}$ ein stetiges Feld von $(n-v)$-Systemen, die zu $M^{v}$ fremd sind, so sagen wir $M^{\nu}$ besitze ein äußeres $(n-\nu)$-Feld $\left.{ }^{25}\right)$. Ist $v=n-1$, so bedeutet dies einfach, daß $M^{v}$ zweiseitig in $M^{n}$ liegt.

Wegen b) ist $M^{v}$ ein Zyklus (mod 2) der $\xi$-Zellenzerlegung, der eine $\nu$-dimensionale Homologieklasse $\Xi^{v}$ von $M^{n}$ und eine $\nu$-dimensionale Homologieklasse $\bar{\Xi}^{v}$ von $M^{\nu}$ repräsentiert. Es gilt:

Satz 23. Besitzt die in $M^{n}$ liegende Hyperfläche $M^{v}$ ein äußeres $(n-v)$ Feld, so ist die Schnittzahl der charakteristischen Klasse $F^{n-\nu}$ von $M^{n}$ mit $M^{v}$ die (mod 2) reduzierte Euler'sche Charakteristik von $\mathbf{M}^{\nu}$.

Bevor wir diesen Satz beweisen, führen wir die folgenden Bezeichnungen ein: $\bar{\xi}$ seien die Zellen der $\xi$-Zellenzerlegung, die nach b) eine Zellenzerlegung der $M^{\nu}$ liefern; ein in der ganzen $M^{n}$ definiertes $(\nu-1)$ dimensionales und aus $(n-\nu+1)$-Systemen bestehendes Gerüst wird mit $\mathfrak{S}$ und der zugehörige Charakter in der Gruppe $\Lambda^{v} \operatorname{der} M^{n}(\S 4, \mathrm{Nr} .4)$ mit $\chi$ bezeichnet. Ein in der ganzen $M^{v}$ definiertes $(\nu-1)$-dimensionales und aus 1-Systemen bestehendes Gerüst wird mit $\overline{\mathscr{S}}$ und der zugehörige Charakter in der Gruppe $\Lambda^{\nu}$ der $M^{v}$ mit $\bar{\chi}$ bezeichnet. Die Charaktere $\chi$ bestimmen in der $v$-dimensionalen Betti'schen Gruppe $\operatorname{der} M^{n} \operatorname{den}$

25) Eine in einer orientierbaren Mannigfaltigkeit liegende Hyperfläche mit åußerem $(n-v) \cdot$ Feld ist orientierbar. 
Charakter $\chi^{\nu}(\S 4$, Nr. 4), während die Charaktere $\bar{\chi}$ in analoger Weise in der $v$-dimensionalen Betti'schen Gruppe von $M^{v}$ den Charakter $\bar{\chi}^{v}$ bestimmen.

Dann beweisen wir den folgenden

Hilfssatz. Gibt es zwei Gerüste $\mathbb{S}$ und $\overline{\mathbb{S}}$, so daß für jede Zelle $\bar{\xi}^{\vee}$ die Relation

$$
\chi\left(\bar{\xi}^{\nu}\right) \equiv \bar{\chi}\left(\bar{\xi}^{\nu}\right) \quad(\bmod 2)
$$

erfüllt ist, so gilt die Behauptung von Satz 23.

Beweis: Durch Summation über alle Zellen $\bar{\xi}^{\nu}$ erhält man aus (2):

$$
\chi^{\nu}\left(\Xi^{v}\right) \equiv \bar{\chi}^{\nu}\left(\bar{\Xi}^{v}\right) . \quad(\bmod 2) .
$$

Die linke Seite von (3) ist nach Satz 16 die Schnittzahl $\varnothing\left(F^{n-\nu}, \Xi^{v}\right)$, während die rechte Seite nach § 5, Formel (3a) der Euler'schen Charakteristik von $M^{\nu}$ kongruent ist. Damit haben wir den Hilfssatz bewiesen.

Um nun noch den Satz 23 zu beweisen, haben wir Gerüste $\mathbb{S}$ und $\overline{\mathfrak{S}}$ zu konstruieren, die der Voraussetzung des Hilfssatzes genügen: Zunächst wird $\overline{\mathcal{S}}$ beliebig gewählt. Auf den Zellen $\bar{\xi}^{\nu-1}$ sollen ferner die Systeme von $\mathbb{S}$ die durch den Vektor von $\mathbb{G}$ ergänzten Systeme des äußeren $(n-\nu)$-Feldes sein; im übrigen Teil der $M^{n}$ wird $\mathfrak{S}$ beliebig unter Verwendung von Satz 14 konstruiert. Durch diese Wahl von $\mathbb{G}$ und $\mathbb{E}$ ist tatsächlich (2) erfüllt, wie man leicht durch Anwendung der Berechnung der Charakteristik durch Rekursion ( $\$ 3, \mathrm{Nr} .2$ ) bestätigt.

Wir gehen nicht auf naheliegende Verallgemeinerungen von Satz 23 ein, sondern wenden diesen Satz nun zur Lösung der am Anfang dieser Nummer gestellten Aufgabe an:

Sat: 24. Zu gegebenem $n$ und $m$ mit $n \equiv m-1(\bmod 2)$ gibt es eine Mannigfaltigkeit $M^{n}$, in der die charakteristische Klasse $F^{m-1}$ nicht die 0-Klasse ist.

Zusatz. Ist $n \equiv m-1(\bmod 4)$, so gibt es sogar eine orientierbare $M^{n}$, in $\operatorname{der} F^{m-1}$ nicht verschwindet.

Zum Beweis dieser Sätze genügen die folgenden Bemerkungen:

1. Die Voraussetzung von Satz 23 ist erfüllt, wenn $M^{n}$ das topologische Produkt aus $M^{v}$ und aus einer beliebigen $(n-v)$-dimensionalen Mannigfaltigkeit ist. 
2. Ist die Voraussetzung von Satz 23 erfüllt, und besitzt $M^{v}$ eine ungerade Euler'sche Charakteristik, so folgt aus diesem Satz, daß in $M^{n}$ die Klasse $F^{n-v}$ nicht verschwindet.

3. Es gibt Mannigfaltigkeiten gerader Dimension mit ungerader Charakteristik, und es gibt orientierbare Mannigfaltigkeiten mit einer durch 4 teilbaren Dimension und ungerader Charakteristik. Nun setze man $m-1=n-v$ und konstruiere $M^{n}$ als Produktmannigfaltigkeit.

Durch spezielle Wahl von $m$ folgt aus dem Zusatz leicht:

Satz 25. Zu jeder von 1 und 3 verschiedenen Dimensionszahl $n$ gibt es eine orientierbare, aber nicht parallelisierbare n-dimensionale Mannigfaltigkeit.

(Man beachte, daß nach Satz 19 das Verschwinden aller charakteristischen Klassen eine notwendige Bedingung für die Parallelisierbarkeit ist.)

\section{Beispiele und Anwendungen}

$x_{0}, x_{1}, x_{2}, \ldots, x_{n}$ seien Koordinaten in einem $(n+1)$-dimensionalen Zahlenraum $R^{n+1}$, und $\mathfrak{p}$ bedeute den Ortsvektor $\left(x_{0}, x_{1}, \ldots, x_{n}\right)$ in diesem Raum. Im $R^{n+1}$ seien $m$ Vektorfelder $\mathfrak{v}^{\mu}$ gegeben. $(\mu=1,2, \ldots, m)$, und für jedes $\mu$ seien die Komponenten $v_{i}^{\mu}(i=0,1,2, \ldots, n)$ des Vektors $\mathfrak{s}^{\mu}$ homogene Funktionen ersten Grades der unabhängigen Variabeln $x_{0}, x_{1}, \ldots, x_{n}$. Diese Vektorfelder projizieren wir vom Nullpunkt des $R^{n+1}$ aus auf den $n$-dimensionalen projektiven Raum $P^{n}$, der den $R^{n+1}$ zum $(n+1)$-dimensionalen projektiven Raum abschließt. Aus unserer Homogenitätsbedingung folgt, daß damit $m$ Vektorfelder im $P^{n}$ definiert sind, die dann und nur dann ein $m$-Feld im Sinne von $\S 4, \mathrm{Nr}$. 1 bilden, wenn die $(m+1)$ Vektoren $\mathfrak{p}=\mathfrak{y}^{0}, \mathfrak{v}^{1}, \mathfrak{v}^{2}, \ldots, \mathfrak{y}^{m}$ in allen Punkten des $R^{n+1}$ außer im Nullpunkt linear unabhängig waren.

Diese bequeme Darstellung der Vektorfelder in projektiven Räumen verwenden wir im folgenden zur Diskussion der charakteristischen Klassen des $n$-dimensionalen projektiven Raumes. So liefern uns zum Beispiel für $n=3$ und $m=3$ die Vektoren

$$
\begin{array}{lrrr}
\mathfrak{v}^{0}\left(x_{0},\right. & x_{1}, & x_{2}, & \left.x_{3}\right) \\
\mathfrak{v}^{1}\left(-x_{1},\right. & x_{0}, & -x_{3}, & \left.x_{2}\right) \\
\mathfrak{v}^{2}\left(-x_{2},\right. & x_{3}, & x_{0}, & \left.-x_{1}\right) \\
\mathfrak{v}^{3}\left(-x_{3},-x_{2},\right. & x_{1}, & \left.x_{0}\right)
\end{array}
$$

im 3-dimensionalen projektiven Raum $P^{3}$ ein stetiges 3-Feld, womit die Parallelisierbarkeit des $P^{3}$ und damit der 3-dimensionalen Sphäre durch ein Beispiel belegt ist. Auch für die Dimensionszahl 7 läßt sich ein analoges Beispiel finden, das den $P^{7}$ und die 7-dimensionale Sphäre parallelisiert ${ }^{26}$ ).

26) vgl. H. Hurwitz: Uber die Komposition der quadratischen Formen von beliebig vielen Variabeln. (Math. Werke, Band II, S. 565-571, speziell S. 570, wo sich die zu I analoge Matrix findet). 
Nun untersuchen wir den Fall $n=5, m=2$, beschäftigen uns also mit 2-Feldern im $P^{5}$. Die drei Vektoren

$$
\begin{aligned}
& \mathfrak{v}^{0}\left(x_{0}, x_{1}, \quad x_{2}, \quad x_{3}, \quad x_{4}, x_{5}\right) \\
& \mathfrak{v}^{1}\left(-x_{1}, x_{0},-x_{3}, \quad x_{2},-x_{5}, x_{4}\right) \\
& \mathfrak{v}^{2}\left(-x_{2}, x_{3}, \quad x_{0},-x_{1}, \quad 0,0\right)
\end{aligned}
$$

sind nur für $x_{0}=x_{1}=x_{2}=x_{3}=0$ linear abhängig, sie liefern also im $P^{5}$ außerhalb der durch $x_{0}=x_{1}=x_{2}=x_{3}=0$ gegebenen projektiven Geraden $P^{1}$ zwei linear unabhängige Vektorfelder, die wir der Einfachheit halber wieder mit $\mathfrak{v}^{1}$ und $\mathfrak{v}^{2}$ bezeichnen. Nun konstruieren wir unter Verwendung der Bezeichnungen von Nr. 2 eine $\xi$-Zellenzerlegung des $P^{5}$, in der der 4-dimensionale projektive Raum $x_{4}=0$ als Hyperfäche $P^{4}$ liegt. Der Schnittpunkt $P$ von $P^{1}$ und $P^{4}$ liege im Innern einer Zelle $\bar{\xi}_{0}^{4}$ der Zellenzerlegung von $P^{4}$. Ferner sollen zwei Gerüste $\mathfrak{G}$ und $\overline{\mathfrak{G}}$ konstruiert werden, die den Voraussetzungen des Hilfssatzes von Nr. 2 genügen: Die Vektoren von $\overline{\mathfrak{G}}$ seien auf den Zellen $\bar{\xi}^{3}$ die Vektoren $\mathfrak{v}^{2}$, während die 2-Systeme von $\mathfrak{G}$ auf den Zellen $\bar{\xi}^{3}$ die Systeme $\mathfrak{y}^{1}, \mathfrak{v}^{2}$ sein sollen; auf den übrigen Zellen $\xi^{3}$ von $P^{5}$ ist $\mathfrak{S}$ beliebig und kann nach Satz 14 konstruiert werden. Die zu $\mathfrak{G}$ und $\bar{G}$ gehörigen Charaktere $\chi$ und $\bar{\chi}$ erfüllen tatsächlich die in der Voraussetzung des Hilfssatzes geforderte Kongruenz (2):

$$
\chi\left(\bar{\xi}^{4}\right) \equiv \bar{\chi}\left(\bar{\xi}^{4}\right) \quad(\bmod 2) .
$$

Zum Beweise beachte man, daß für jede Zelle $\bar{\xi}^{4}$ außer $\bar{\xi}_{0}^{4}$ die Relation $\chi\left(\bar{\xi}^{4}\right)=\bar{\chi}\left(\bar{\xi}^{4}\right)=0$ besteht, da sowohl $\mathbb{S}$ als auch ${ }^{\mathbb{S}}$ ins Innere der Zelle stetig fortgesetzt sind. Für die Zelle $\bar{\xi}_{0}^{4}$ bestätigt man die Behauptung durch Berechnung der Charakteristik durch Rekursion ( $\$ 3, \mathrm{Nr} .2$ ); um diese Methode anwenden zu können, genügt es, daß die Zelle $\bar{\xi}_{0}^{4}$ zum projektiven Raum $x_{5}=0$ fremd ist; dann liegen die Vektoren $\mathfrak{v}^{1}$, deren Angriffspunkte Punkte von $\bar{\xi}_{0}^{4}$ sind, nicht in $P^{4}$.

Aus der Behauptung des Hilfssatzes folgt nun, daß die Schnittzahl der Klasse $F^{1}$ des $P^{5}$ mit der Hyperfläche $P^{4}$ die $(\bmod 2)$ reduzierte Charakteristik von $P^{4}$ ist; diese Charakteristik hat aber den Wert 1 . Somit ist die Klasse $F^{1}$ nicht die 0-Klasse und wird durch eine projektive Gerade repräsentiert.

Mit Hilfe von analogen Vektorfeldern gelingt die Bestimmung der Klasse $F^{1}$ in projektiven Räumen der Dimension $4 k+1(k>0)$; man findet:

Satz 26. Die eindimensionale charalkteristische Klasse in einem reellen projektiven Raum der Dimension $(4 k+1)(k>0)$ wird durch eine projektive Gerade repräsentiert; es ist daher unmöglich, in diesen Räumen zwei linear unabhängige stetige Vektorfelder zu finden.

\section{Eine algebraische Anwendung}

Wir wollen unsere Untersuchung der projektiven Räume mit einem algebraischen Problem in Beziehung setzen, das in engem Zusammenhang mit älteren Untersuchungen steht ${ }^{27}$ ).

27) vgl. Hurwitz: Werke, Band II, S. 565-571 und S. 641-666; ferner Radon: Abh. math. Seminar der Univ. Hamburg, Band I, S. 1-14. 
Wir nennen $(m+1)$ linear unabhängige quadratische $(n+1)$-reihige reelle Matrizen

$$
A^{(\mu)}=\left(a_{i k}^{\mu}\right),\left(\begin{array}{c}
\mu=0,1,2, \ldots, m \\
i, k=0,1,2, \ldots, n
\end{array}\right)
$$

linear unabhängig, wenn jede durch Linearkombination entstandene Matrix $\Sigma A^{(\mu)} y_{\mu}$ nicht-singulär ist, sobald nur eine der reellen Zahlen $y_{\mu}$ von 0 verschieden ist. Dann gilt der folgende

Hilfssatz: Gibt es $(m+1)$ linear unabhängige Matrizen (1), so existiert im projektiven Raum $P^{n}$ ein überall stetiges $m$-Feld.

Beweis: Ist $B$ irgend eine nicht-singuläre $(n+1)$-reihige Matrix, so sind offenbar auch die Matrizen $B A^{(\mu)}(\mu=0,1, \ldots, m)$ linear unabhängig; da wir $B=\left(A^{(0)}\right)^{-1}$ wählen können, dürfen wir daher von vorneherein voraussetzen, daß:

$$
a_{i k}^{0}=\left\{\begin{array}{l}
0 \text { für } i \neq k \\
1 \text { für } i=k
\end{array}\right.
$$

ist. Wir verstehen nun für $\mu=0,1, \ldots, m$ unter $\mathfrak{y}^{\mu}$ den Vektor des $R^{n+1}$, dessen $i$-te Komponente $(i=0,1, \ldots, n)$ durch

$$
v_{i}^{\mu}=\sum_{k=0}^{n} a_{i k}^{\mu} x_{k}
$$

gegeben ist; dann ist $\mathfrak{v}^{0}$ mit Rücksicht auf (2) der Ortsvektor $\mathfrak{p}=\left(x_{0}, x_{1}, \ldots\right.$, $x_{n}$ ) im $R^{n+1}$. Aus Nr. 3 folgt, daß die Behauptung des Hilfssatzes bewiesen ist, sobald gezeigt ist, daß die $(m+1)$ Vektoren $\mathfrak{y}^{\mu}$ für $\mathfrak{p} \neq 0$ linear unabhängig sind.

Es sei also $\sum_{\mu=0}^{m} y_{\mu} \mathfrak{y}^{\mu}=0$ für einen gewissen Vektor $\mathfrak{p} \neq 0, \mathrm{~d}$. h.

$$
\sum_{k, \mu} a_{i k}^{\mu} x_{k} y_{\mu}=0, \quad(i=0,1,2, \ldots, n) ;
$$

da $\mathfrak{p} \neq 0$ ist, ist der Rang der Matrix $\left(\sum_{\mu} a_{i k}^{\mu} y_{\mu}\right)$ kleiner als $(n+1)$; da die Matrizen $A^{(\mu)}=\left(a_{i k}^{\mu}\right)$ linear unabhängig sind, ist dies nur möglich, wenn alle $y_{\mu}=0$ sind. Das war zu beweisen.

Der Hilfssatz gestattet nun die folgende algebraische Formulierung des Satzes 26:

Satz 27. Je drei quadratische $(4 k+2)$-reihige Matrizen sind linear abhängig $(k \geqq 0)$. 


\section{Die eindimensionale charakteristische Klasse in orientierbaren dreidimensionalen Mannigfaltigkeiten}

Wir haben in $\S 5$, Nr. 3 gesehen, daß in einer dreidimensionalen Mannigfaltigkeit $M^{3}$ das Verschwinden der charakteristischen eindimensionalen Klasse $F^{1}$ eine notwendige und hinreichende Bedingung für die Parallelisierbarkeit ist; wir haben ferner erwähnt, daß $F^{1}$ in einer orientierbaren $M^{3} \mathrm{mit}$ differenzierbarer Simplizialzerlegung immer die 0-Klasse ist, sind aber dem Leser den Beweis dieser Tatsache schuldig geblieben; er soll jetzt unter etwas anderen Differenzierbarkeitsvoraussetzungen nachgeholt werden.

\section{Ein kombinatorisches Lemma}

Das folgende Lemma ist an sich interessant und nützlich für das Studium dreidimensionaler Mannigfaltigkeiten.

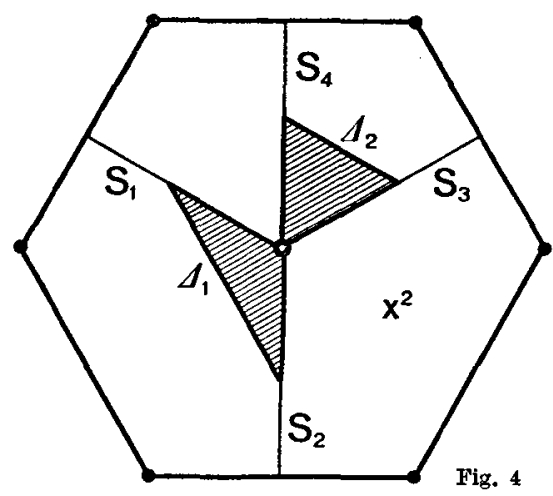

Lemma: Jede Zellenzerlegung einer dreidimensionalen Mannigfaltigkeit $M^{3}$ läßt sich zu einer solchen Unterteilung $U$ verfeinern, da $B$ jede zweidimensionale Homologieklasse $(\bmod 2)$ von $M^{3}$ durch einen Teilzyklus von $U$ repräsentiert werden kann, der aus einer oder mehreren zueinander fremden zweidimensionalen Mannigfaltigkeiten besteht.

Es ist also zu zeigen, daß es zu jedem zweidimensionalen Zyklus $z^{2}$ der gegebenen Zellenzerlegung in $U$ eine oder mehrere fremde Flächen gibt, die in ihrer Gesamtheit einen zu $z^{2}$ homologen Zyklus bilden. Der Beweis erfolgt in zwei Schritten:

1. $z^{2}$ ist ein Zyklus ( $\left.\bmod 2\right)$, in jeder Kante von $z^{2}$ stoßen also eine geradeZahl von Polygonen von $z^{2}$ zusammen. Wir betrachten nun eine Kante $\xi^{1}$ von $z^{2}$, in der mehr als zwei (etwa $2 n$ ) Polygone zusammenstoßen. $\xi_{1}^{0}$ und $\xi_{2}^{0}$ seien die Randpunkte von $\xi^{1}$, und $x^{2}$ die duale Zelle zu $\xi^{1}$ in der gegebenen Zellenzerlegung der $M^{3}$. Die Schnittstrecken von $x^{2}$ mit den $2 n$ in $\xi^{1}$ zusammenstoßenden Polygonen bezeichnen wir mit $s_{1}, s_{2}, \ldots, s_{2 n}$, wobei die Numerierung durch die natürliche zyklische Ordnung dieser Strecken gegeben sein soll (siehe Fig. 4 für $n=2$ ). Zwischen zwei aufeinanderfolgende Strecken 
$s_{2 k-1}$ und $s_{2 k}(k=1,2, \ldots, n)$ schalten wir nun ein kleines Dreieck $\Delta_{k}$ ein (Fig. 4) und konstruieren den Kegel $K_{k 1}$ über dem Rand von $\Delta_{k}$ mit der Spitze $\xi_{1}^{0}$. Analog wird $K_{k 2}$ mit der Spitze $\xi_{2}^{0}$ konstruiert. $K_{k 1}+K_{k 2}$ ist ein zweidimensionaler 0-homologer Zyklus, also $z^{2}+\Sigma\left(K_{k 1}+K_{k 2}\right)$ ein $z u z^{2}$ homo-

loger Zyklus, in dem $\xi^{1}$ durch Kanten ersetzt ist, von denen jede mit genau zwei Polygonen dieses neuen Zyklus inzident ist. Zur Durchführung dieser Konstruktion ist natürlich eine geeignete Unterteilung der gegebenen Zellenzerlegung einzuführen.

Werden durch diese Konstruktion alle Kanten von $z^{2}$, in denen mehr als zwei Polygone zusammenstoßen, beseitigt, so erhält man einen zu $z^{2}$ homologen Zyklus $\bar{z}^{2}$, der aus einer oder mehreren zueinander fremden Pseudomannigfaltigkeiten besteht.

2. Sei $\xi^{0}$ eine beliebige Ecke von $\bar{z}^{2}$. Wir konstruieren eine Unterteilung $U$, in der die Sterne der Ecken $\xi^{0}$ zueinander fremd sind. $S^{2}$ sei die Randsphäre des Sternes von $\xi^{0}$. Der Schnitt von $\bar{z}^{2}$ mit $S^{2}$ besteht aus einigen zueinander fremden einfach geschlossenen Polygonumfängen, die einen Teilkomplex $C^{2}$ von $S^{2}$ beranden. Uber dem Rand von $C^{2}$ konstruieren wir den Kegel $K^{2}$ mit der Spitze $\xi^{0} ; C^{2}+K^{2}$ ist ein 0-homologer zweidimensionaler Zyklus, also $\bar{z}^{2}+C^{2}+K^{2}$ ein zu $\bar{z}^{2}$ homologer Zyklus, durch den wir $\bar{z}^{2}$ ersetzen.

Führt man diese Konstruktion für jede Ecke $\xi^{0}$ durch, so entsteht ein zu $\bar{z}^{2}$ und also auch zu $z^{2}$ homologer Zyklus, der aus einigen fremden zweidimensionalen Flächen besteht.

\section{Bestimmung der Klasse $F^{1}$}

Wir bestimmen nun die Klasse $F^{1}$ einer vorgegebenen orientierbaren Mannigfaltigkeit $M^{3}$, indem wir $M^{3}$ mit einer ,Standardmannigfaltigkeit“" $\boldsymbol{M}_{0}^{3}$ vergleichen. $\boldsymbol{M}_{0}^{3}$ ist entweder der dreidimensionale projektive Raum $\boldsymbol{P}^{3}$ oder das topologische Produkt $T^{3}=S^{2} \times S^{1}$ aus einer Sphäre und einer Kreislinie. Beide Standardmannigfaltigkeiten sind parallelisierbar. (Die Parallelisierbarkeit von $P^{3}$ wurde in $\S 6, \mathrm{Nr} .3$ bewiesen; wegen Satz 23 ist in $T^{3}$ die Klasse $F^{1}$ die 0-Klasse, also ist $T^{3}$ parallelisierbar. Man kann übrigens auch direkt ein stetiges 3-Feld in $T^{3}$ angeben.) Die gegebene Mannigfaltigkeit $M^{3}$ erfülle nun die folgende Voraussetzung:

Jede in die $\boldsymbol{M}^{3}$ singularitätenfrei eingebettete zweidimensionale Mannigfaltigkeit besitzt eine Umgebung, die sich topologisch und stetig differenzierbar in eine der Standardmannigfaltigkeiten abbilden läßt.

Diese Voraussetzung ist nur eine Differenzierbarkeitsvoraussetzung, denn jede in die $M^{3}$ singularitätenfrei eingebettete zweidimensionale Mannigfaltigkeit $F$ besitzt eine Umgebung, die sich topologisch in eine der Standardmannigfaltigkeiten abbilden läßt. Um dies zu zeigen, konstruiere man in $P^{3}$ oder $T^{\mathbf{3}}$ singularitätenfrei eine zu $F$ homöomorphe Mannigfaltigkeit $F^{\prime}$. (Bei der Durchführung dieser Konstruktion sind drei Fälle zu unterscheiden: a) $F$ ist orientierbar; dann kann $F^{\prime}$ in $P^{3}$ oder in $T^{3}$ konstruiert werden. b) $F$ ist nicht orientierbar und besitzt eine ungerade Euler'sche Charakteristik; dann kann $F^{\prime}$ in $P^{3}$ konstruiert werden. c) $F^{\prime}$ ist nicht orientierbar und besitzt gerade Charakteristik; dann kann $F^{\prime}$ in $T^{3}$ konstruiert werden.) Da nun $M^{3}$ orientier- 
bar ist, liegt $F^{\prime}$ zweiseitiges) in $M_{0}^{3}$, sobald $F^{\prime}$ zweiseitig in $M^{3}$ liegt, und ebenso liegt $F^{\prime}$ einseitig in $M_{0}^{3}$, wenn $F$ einseitig in $M^{3}$ liegt; eine topologische $\mathrm{Ab}$ bildung von $F$ auf $F^{\prime}$ kann also immer zu einer topologischen Abbildung einer Umgebung von $F$ auf eine Umgebung von $F^{\prime}$ ergänzt werden. Damit ist unsere Behauptung bewiesen.

Nun betrachten wir die im Lemma erwähnte Zellenzerlegung $U$ der $M^{3}$, deren Zellen wir mit $\xi^{r}$ bezeichnen; ferner sei $F$ nun im speziellen ein Teilzyklus (mod 2) von $U$, der aus den Zellen $\bar{\xi}^{s}$ von $U$ bestehen soll. Denken wir uns in der Standardmannigfaltigkeit $M_{0}^{3}$ ein stetiges 2-Feld konstruiert, so liefert die nach der Voraussetzung existierende Abbildung einer Umgebung von $F$ in die $M_{0}^{3}$ in dieser Umgebung ein stetiges 2-Feld $\mathscr{F}$. Die in den Punkten der Zellen $\bar{\xi}^{1}$ angreifenden 2 -Systeme von $\mathscr{F}$ bilden ein eindimensionales Gerüst ( $\$ 4, \mathrm{Nr} .2$ ), das nach Satz $14 \mathrm{zu}$ einem in der ganzen $M^{3}$ definierten eindimensionalen und aus 2-Systemen bestehenden Gerüst $\mathfrak{G}$ ergänzt werden kann. Der zu $\mathfrak{G}$ gehörige Charakter $\chi(\S 4, \mathrm{Nr} .4)$ hat für jede Zelle $\bar{\xi}^{2}$ den Wert 0 , denn das durch $\mathbb{S}$ auf $\dot{\xi}^{2}$ gelieferte 2-Feld ist stetig ins Innere von $\bar{\xi}^{2}$ fortgesetzt. Es ist also $\chi(F)=0$, mit anderen Worten: Die charakteristische Klasse $F^{\mathbf{1}}$ hat mit $F$ die Schnittzahl 0 . Da nun $F^{1}$ mit jeder Fläche $F$ die Schnittzahl 0 hat, und andererseits nach unserem Lemma jede zweidimensionale Homologieklasse $(\bmod 2)$ durch eine oder mehrere zweidimensionale Mannigfaltigkeiten $F$ repräsentiert werden kann, hat $F^{1}$ mit jeder zweidimensionalen Homologieklasse die Schnittzahl 0, ist also nach dem Poincaré-Veblen'schen Dualitätssatz die 0-Klasse $(\bmod 2)$.

\section{A N H A N G II}

\section{Über die Darstellung von Hyperflächen im euklidischen Raum durch ein System von Gleichungen ${ }^{29}$ )}

In diesem Anhang ziehen wir aus dem Schnittsatz 23 eine Folgerung. In Analogie zu $\S 6$, Nr. 2 verstehen wir unter einer $v$-dimensionalen, in den $n$-dimensionalen euklidischen Raum $R^{n}$ eingebetteten Hyperfläche einen Teilkomplex einer Zellenzerlegung des $R^{n}$, der das topologische Bild einer $\nu$-dimensionalen Parametermannigfaltigkeit vermöge einer topologischen und stetig differenzierbaren Abbildung ist. $(1<v<n)$.

Seien nun $x_{1}, x_{2}, \ldots, x_{n}$ cartesische Koordinaten im $R^{n}$, und seien $(n-\nu)$ stetig differenzierbare Funktionen $f_{i}\left(x_{1}, x_{2}, \ldots, x_{n}\right)(i=1,2, \ldots, n-\nu)$ dieser Koordinaten gegeben. Definieren nun die Gleichungen

$$
f_{i}\left(x_{1}, x_{2}, \ldots, x_{n}\right)=0
$$

eine $\nu$-dimensionale Hyperfläche $M^{\nu}$, und besitzt die Funktionalmatrix der Funktionen $f_{i}$ in jedem Punkt der $M^{v}$ den Rang $(n-v)$, so nennen wir $M^{v}$ eine „,durch Gleichungen regulär darstellbare Hyperfläche“s.

28) Über die Beziehungen zwischen den Begriffen ,orientierbar" und „,zweiseitig“ vgl. Seifert-Threlfall, $\$ 76$.

29) Dieser Anhang ist nachträglich als Antwort auf eine Frage von Herrn H. Seifert entstanden. 
Satz 28. Jede durch Gleichungen regulär darstellbare Hyperfläche besitzt eine gerade Euler'sche Charakteristik.

Beweis: Die Gradienten grad $f_{i}$ der Funktionen $f_{i}$, die in den Punkten von $M^{\nu}$ angreifen, sind fremd zu $M^{\nu}(\S 6, \mathrm{Nr} .2)$ und die in einem Punkte der $M^{\nu}$ angreifenden Gradienten sind nach Voraussetzung linear unabhängig, bilden also ein $(n-v)$-System. Da diese Systeme überdies stetig mit ihrem Angriffspunkt variieren, besitzt $M^{\nu}$ ein äußeres $(n-v)$-Feld im Sinne von $\S 6, \mathrm{Nr} .2$.

Nun schließen wir den euklidischen Raum $R^{n}$ durch einen unendlich fernen Punkt zur $n$-dimensionalen Sphäre $S^{n}$ ab. Unsere in $S^{n}$ liegende Hyperfläche $M^{\nu}$ erfüllt die Voraussetzung von Satz 23, ihre Charakteristik ist also nach diesem Satz $(\bmod 2)$ der Schnittzahl der charakteristischen Klasse $F^{n-\nu}$ der $S^{n}$ mit $M^{\nu}$ kongruent. $\mathrm{Da} F^{n-\nu}$ in $S^{n}$ trivialerweise die 0-Klasse ist, verschwindet diese Schnittzahl, womit unsere Behauptung bewiesen ist.

Aus Satz 28 folgt im speziellen, daß in keinem euklidischen Raum beliebiger Dimension eine zur reellen oder komplexen projektiven Ebene homöomorphe und durch Gleichungen regulär darstellbare Hyperfläche liegt ${ }^{30}$ ).

\footnotetext{
$\left.{ }^{30}\right)$ Die Euler'sche Charakteristik der reellen projektiven Ebene ist 1, die der komplexen projektiven Ebene 3 (vgl. B. L. van der Waerden: Topologische Begründung des Kalküls der abzählenden Geometrie. Math. Ann. 102, 1929, S. 337-362, speziell S. 361). Daß die reelle projektive Ebene in keinem $R^{n}$ durch Gleichungen regulär darstellbar ist, folgt aus dem allgemeineren Satze, daß jede im $R^{n}$ durch Gleichungen regulär darstellbare Mannigfaltigkeit orientierbar ist. (Für den Beweis vgl. die Fußnote 25). Dieser Satz wurde schon von Poincaré bewiesen. (J. Ec. pol. 2 e série, t. I, p. 33). Die im Buche von Hilbert und Cohn-Vossen über anschauliche Geometrie (Berlin, J. Springer, 1932) auf S. 301 gegebene Darstellung der projektiven Ebene im $R^{4}$ ist nicht regulär.
} 


\section{LEBENSLAUF}

In Zürich, wo ich am 21. April 1909 geboren wurde, habe ich die Primarschule und in den Jahren 1921 bis 1927 das kantonale Realgymnasium bis zum Abschluß durch die Maturitätsprüfung besucht. Die Fortsetzung meiner Ausbildung übernahm die Eidgenössische Technische Hochschule, deren IX. Abteilung ich von 1927 bis zur Diplomprüfung im Jahre 1931 als Studierender angehört habe, und seit 1932 als Assistent noch angehöre.

Nach einjährigem Studium in Deutschland (Wintersemester 1931/32 in Hamburg, Sommersemester 1932 in Göttingen) wurde ich Assistent für darstellende Geometrie bei Herrn Prof. W. Saxer; im Sommersemester 1934 habe ich meine jetzige Stellung als Assistent am mathematischen Seminar erhalten. Ferner durfte ich im Wintersemester 1932/33 bei Herrn Prof. G. Polya in höherer Mathematik assistieren.

1933-35 war ich Hilfslehrer für darstellende Geometrie am kantonalen Gymnasium in Zürich.

Herrn Prof. H. Hopf bin ich für die Anregung und Förderung dieser Arbeit zu großem Dank verpflichtet. 